JOURNAT, OF MATHFMATICAL, PSYCHOLOGY: 5, 1-48 (1968)

\title{
A Theory of Context Effects Based on Cross-Context Matching ${ }^{1}$
}

\author{
David H. KRaNTZ
}

University of Michigan, Ann Arbor, Michigan 48104

\begin{abstract}
In cross-context matching, an observer reports that some stimulus elements, seen in one context, match other stimulus elements, see in a different context. The effect of changing from context $S$ to context $T$ defines a function $g_{S, T}$, where $g_{S, T}(A)=B$ if stimulus $A$ in $T$ matches $B$ in $S$.

The description of context changes by functions is particularly powerful when there exist a semigroup of transformations of the stimulus elements that exhibits a special property called context-invariance. In this case, the functions $g_{S, T}$ are affine transformations of commutative groups. This means that knowledge of some effects of a context change can be used, via the group structure, to predict other effects. Predictive power is increased further when the contexts themselves are related by transformations that leave cross-context matching invariant; and the greatest power is obtained when stimuli and contexts have vector structure, as with color stimuli. Some previous theories of context effects in color are discussed from the standpoint of different semigroups of context-invariant transformations.
\end{abstract}

\section{INTRODUCTION}

The perceived qualities of an object depend not only on those features of the proximal stimulus that are directly influenced by the physical features of the object, but also on the immediate spatial and temporal context in which the object is embedded. In some instances, it is possible to study the effects of context by a method of cross-context matching. This means that an observer is able to report that some stimulus elements, in one context, match other stimulus elements, in another context, with respect to certain subjective dimensions. From such reports of identical phenomenal qualities, we can construct, for any pair of contexts, a pairing of stimulus elements. If $A, B$, denote stimulus elements, and $S, T$, denote contexts, the notation $A^{T} \equiv B^{S}$ will mean " $A$, in context $T$, matches $B$, in context $S$ (with respect to the

1 The research on which this paper is based was begun while the author was a National Science Foundation Predoctoral Fellow at the University of Pennsylvania. It was supported in part by a grant-in-aid of research from Sigma XI-RESA research funds. Preparation of the manuscript was aided by funds from NSF grant G-17637 to the University of Pennsylvania, and from NIMH grant MH 11504-01 to the University of Michigan. The author is grateful to Leo M. Hurvich, Dorothea Jameson Hurvich, and R. Duncan Luce for helpful criticisms of early versions of the paper.

(C) 1968 by Academic Press Inc.

$480 / 5 / \mathrm{x}-\mathrm{I}$ 
specified subjective dimensions)." Corresponding to contexts $S, T$, one has the set of all pairs $A, B$, such that $A^{T} \equiv B^{S}$.

The most clear-cut example of cross-context matching, which is also the example that has guided the formal theory to be presented in this paper, comes from the field of color vision. Here, a stimulus element consists of a portion of the visual field which is homogeneous with respect to color; the context can be varied by keeping all viewing conditions constant except for the makeup of a preadapting or a surrounding field. Cross-context matching can be carried out by a simultaneous or successive viewing of two stimulus elements in different contexts (some considerations regarding the technique used will be mentioned below). The stimulus elements can be matched with respect to color (hue, saturation, and brightness) or with respect to only some dimensions of color (only the brightness dimension has been studied systematically in isolation from the others). Quite a different example involves the matching of two similar objects, seen in different contexts, with respect to subjective size or distance or or both.

Of course, not all context effects can be studied by cross-context matching. Sometimes contexts cannot be manipulated back and forth-for example, it might be difficult to erase memory in order to study memory color by this technique. In other instances, it may not be possible to isolate a stimulus element whose appearance changes with changing context-one might hesitate, for example, to match the attractiveness of female noses where the variations in context involve different curvatures of lips and eyebrows.

Nevertheless, in some instances, cross-context matching may provide a valuable description of the changes of appearance due to changes of context. The description takes the form of a relation, or pairing, on the stimulus elements, as specified above. This sort of description immediately suggests a mathematical question: What theorems can be derived from the formal properties of the pairing? For example, one formal property that may hold approximately for such pairings is transitivity: If $A^{U} \equiv B^{T}$ and $B^{T} \equiv C^{S}$, then $A^{U} \equiv C^{S}$. (This property really involves three pairings, for the contexts $T, U$, and $S, T$, and $S, U$.) Other formal properties may be specific to the domain of stimulus elements and the dimensions on which matching takes place. One of the tasks of experimental analysis is to establish interesting properties of these pairings.

In subsequent sections of this paper, a mathematical theory based on formal properties of cross-context pairings is presented. The fundamental assumptions of the theory involve the concept of a family of transformations of the stimulus elements. A transformation of the stimulus elements is a function that assigns to each stimulus element under consideration another stimulus element, in a well-defined manner. Transformations will be denoted by lowercase letters $a, b$, etc. The notation $A^{a}$ will be used to denote the stimulus element which corresponds to $A$ under the transformation $a$. For examples of transformations, we turn to color stimuli. If the stimulus elements are single portions of the visual field that are homogeneous with respect 
to color, one simple type of transformation involves additive mixture of wavelength distributions. Specifically, let each stimulus element $A$ correspond to a certain spectral energy distribution function (specifying the energy density at each wavelength of the visible spectrum), the size, shape, position, etc. of the homogeneous element of the visual field being held constant. Then a particular transformation of this set of stimulus elements might involve addition of a constant spectral energy distribution to each stimulus element. One way to realize the transformation physically would be to use two projectors to provide homogeneous illumination of the same spot on a diffusing screen. One projector would provide the various untransformed stimulus elements, while the other projector, when turned on, would transform each stimulus element by addition of a fixed spectral distribution. The transformation could be changed by changing the spectral energy distribution added by the second projector. A different sort of transformation of the stimuli would involve increasing the energy of each stimulus just enough to provide a fixed increment in subjective brightness. In this case, for any stimulus elements $A$ and $B$, the perceived brightness differences between $A^{a}$ and $A$, and $B^{a}$ and $B$, would be identical. This sort of transformation could never be achieved by adding a fixed spectral distribution from a second projector. (Even if all the stimulus elements had the same shape wavelength distribution, fixed increments in energy would not lead to fixed increments in brightness, according to Stevens' [1957] psychophysical law.) A third example of a transformation of stimulus elements involves stimulus clements consisting of two adjacent homogencous portions of the visual field. Either of the above two transformations could be applied to just one portion, leaving the spectral distribution of the other portion as it was; this would still constitute a transformation of the stimulus elements, considered as pairs of subelements.

The above examples are intended to make clear the generality of the notion of transformation of stimulus elements, as well as to give examples of transformations that might actually be of interest in color vision. The essential feature is that for each element $A$ under consideration, the transformation yields a definite element $A^{a}$.

The first type of transformation mentioned above involves a notion of additive combination of stimulus elements. Thus, it requires the existence of some prior structure (in this case, additive structure) on the stimulus elements. The same type of transformation may be useful in any situation where a useful additive structure exists.

One advantage of considering transformations is that, even if the set of stimulus elements possesses no structure, a family of transformations might be constructed so as to possess some structure. Given two transformations $a$ and $b$, one can take any stimulus element $A$ and apply $a$, obtaining a new element $A^{a}$. In turn, $b$ can be applied to $A^{a}$, yielding a third element $\left(A^{a}\right)^{b}$, or $A^{a b}$. This shows that from any two transformations, one can form a product $a b$, which assigns to any element $A$ the new element $A^{a b}=\left(A^{a}\right)^{b}$. If $a$ and $b$ are of any of the types of transformations mentioned above as examples, then $a b$ is another transformation of that same type. A family of trans- 
formations such that the product of any two transformations in the family is again in the family is called a semigroup of transformations (the associative law, $(a b) c=-a(b c)$, is automatically true). A semigroup of transformations possesses a multiplicative structure and is thus a useful starting point for a "rich" theory of formal properties.

In the case of transformations of color stimuli by means of addition of a fixed spectral distribution, performing two such transformations in sequence involves adding the sum of the two fixed distributions to the original distribution. 'Thus, the product structure on the transformations is just the additive structure of the stimulus elements; nothing really new is introduced by considering transformations. As will be shown, this feature can be generalized: from the product structure on transformations, one can, under suitable conditions, define an additive structure on stimulus elements, such that each transformation corresponds simply to addition of a fixed element.

Aside from formal considerations of structure, which make it reasonable to use a semigroup of transformations of the stimulus elements as a starting point for theory, there are some empirical considerations as well. A transformation $a$ produces a certain kind of change in appearance under a context $T$, namely, the change from the appearance of $A^{T}$ to the appearance of $A^{a T}=\left(A^{a}\right)^{T}$. On matching $A^{T}$ and $A^{a T}$ in context $S$, we find the same change in appearance in going from $B^{S}$ to $C^{S}$, that is, we have the relations

$$
A^{T} \equiv B^{S}, \quad A^{a T} \equiv C^{S} .
$$

Thus the transformation $a$ induces a certain pairing of stimulus elements, namely the pairing between an element $B$ that matches the appearance of an untransformed element, and an element $C$ that matches the appearance of its transform. It seems reasonable, in studying context effects, to try to discover a semigroup of transformations such that the above pairing, induced by a transformation $a$ in the semigroup, corresponds to another transformation $b$ of the same family. That is, whenever $A^{T} \equiv B^{S}$ and $A^{a T} \equiv C^{S}$, then for some $b$ in the same family, $B^{b}=C$. The transformation $b$ must depend on $a$, and, of course, on $S$ and $T$, but should be independent of the choice of $A$. That is, we should try to find a semigroup of transformations such that all the changes in appearance produced under context $T$ by any one transformation correspond to changes in appearance produced under $S$ by another transformation of the same semigroup. That is, such a semigroup of transformations represents a context-invariant type of change in appearance, at least for contexts $S, T$. Below, we will call such a semigroup a context-inzariant (with respect to $S, T$ ) semigroup of transformations, except that we will not necessarily require that there always be a transformation such as $b$ above, taking $B$ into $C$, rather, only that $B$ and $C$ can be taken into the same element by a pair of suitably chosen transformations in the semigroup, i.e., $B^{b}=C^{c}$ for some $b, c$.

Methods for discovering context-invariant semigroups of transformations will not be discussed here. Clearly the semigroup, if it can be discovercd at all, will vary with 
the type of context-shift being considered. Discovery of such transformations appears to be a reasonable subgoal of empirical research on context effects, and in any case, the mathematical theory to be presented starts with the assumption that an appropriate context-invariant semigroup of transformations has been found. Thus, the general theory is to some extent programmatic, although for color vision, specific semigroups will be considered.

In color vision consideration of transformations opens up extra possibilities for cross-context matching. For instance, suppose that one set of viewing conditions involves a homogeneous field of light viewed through an aperture in a light-tight booth. No spectral energy distribution for the homogeneous field will yield a dark brown or pitch black appearance. These colors may be obtained, however, by introducing a bright surround about the aperture. Thus, an energy distribution which yields a brown or black appearance with a bright surround (context $T$ ) cannot be paired with any stimulus element yielding the same appearance in context $S$, the dark surround. However, one may hope to transform such a stimulus clement into one which can be matched in context $S$. If the transformation used is a member of an $S, T$-context-invariant semigroup, one can find the corresponding transformation which produces, in context $S$, the same changes in appearance as are produced by the first transformation in context $T$. One can then apply the "inverse" of the context $S$ transformation to obtain at least a formal "matching element" for the original stimulus element. By this extension of cross-context matching, adaptation and contrast effects can be represented by transformations of "color space." Of course, the form of these transformations will depend on the form of the transformations in the $S, T$-invariant semigroup. If the semigroup of additive transformations (the first example cited above) is $S, T$-invariant, then, as will be shown in Sec. 8 , the formal elements are vectors in "color space." These ideas generalize to domains other than color: under the assumptions below, context changes can be represented as transformations of an "appearance space."

Sections 2-7 present the general theory of context effects based on context-invariant semigroups. This theory is developed with few special assumptions about the nature of the stimulus domain. Special applications to color are considered in Sec. 8. For this purpose some preliminary material on color mixing and color matching is first presented. This material includes a canonical definition of "color space" based on the formal properties of color matching, which has not previously appeared in the color literature. Sections 9 and 10 review some previous work on color adaptation and contrast in relation to the present theory.

\section{PRELIMINARY MATERIAL ON SEMIGROUPS}

This section presents some preliminary, purely mathematical material concerning semigroups and semigroups of transformations. Although elementary, this material 
is essential for subsequent sections, and it seems better to present it here, with only a brief indication of how it is to be used, than to disrupt later sections with expositions of elementary abstract algebra.

Let $X$ be a set, with elements $x, y$, ctc. The set $X$ is a semigroup if there is a binary operation that assigns to every ordered pair $(x, y)$ of elements of $X$ a third element, denoted $x y$, in such a way that the associative law holds: for all $x, y, z$,

$$
(x y) z=x(y z) .
$$

Sometimes the binary operation is denoted by + and the third element assigned to $(x, y)$ is correspondingly denoted $x+y$.

If the elements of $X$ are transformations of a set $K$ into itself (so that to each element $k$ of $K$ and to each $x$ in $X$ there corresponds a transformed element, denoted $k^{x}$, in $K$ ), then the usual binary operation assigns to the pair $(x, y)$ the transformation of $K, x y$, that transforms $k$ into $k^{x y}=\left(k^{x}\right)^{y}$; that is, first transform $k$ by $x$, then transform the result by $y$. For this binary operation, the associative law is automatically satisfied, so that the only constraint in asserting that a set of transformations is a semigroup comes from the necessity that for any $x, y$ in the set, the transformation $x y$ is again a member of the same set.

A semigroup $X$ is commutative if for all $x, y$ in $X, x y=y x$.

A semigroup $X$ is a cancellation semigroup if for all $x, y, z$ in $X$, if either $x z=y z$ or $z x=z y$, then $x=y$.

For a semigroup of transformations, commutativity simply means that the order in which the transformations are applied is irrelevant. If $X$ is a commutative semigroup of transformations of $K$, such that all the transformations are one-to-one $\left(k^{x} \neq l^{x}\right.$ if $k \neq \ell$ ), then $X$ is a cancellation semigroup: for if $x z=y z$, then for any $k$ in $K$, $k^{x z}=k^{y z}$, and since $z$ is one-to-one, $k^{x}=k^{y}$. Thus, $x$ and $y$ have identical effects on every element of $K$, so $x=y$ (transformations are equal when they have identical effects). Similarly, if $z x=z y$, then by commutativity, $x z=y z$, so $x=y$.

An identity in a semigroup $X$ is an element $z$ such that for every $x, x z=x=z x$. If $z$ is an identity in $X$, then an element $y$ is called an inverse of $x$ with respect to $z$ if $x y=z=y x$. A semigroup which has an identity, with respect to which every element has an inverse, is called a group. A group always satisfies cancellation, as can easily be shown.

If $X$ is a commutative cancellation semigroup, it can always be identified as a part of a larger group. To construct such a group, form pairs $(x, y)$ of elements of $X$, where the pair is to be interpreted as a difference or a ratio of $x$ to $y$. For example, the positive integers form a commutative cancellation semigroup, with ordinary multiplication as the binary operation. They are not a group, since inverses of form $1 / 2$, $1 / 3$, etc. are fractions, not integers; but the integers are part of the multiplicative group of positive rational numbers. Each rational number is identified with a class of pairs $\mathrm{m} / \mathrm{n}$; for example, $1 / 2$ and $2 / 4$ define the same number, and in general, $m_{1} / n_{1}$ 
and $m_{2} / n_{2}$ define the same number when $m_{1} n_{2}=m_{2} n_{1}$. Similarly, for the general case, pairs $\left(x_{1}, y_{1}\right)$ and $\left(x_{2}, y_{2}\right)$ are equivalent [notation: $\left(x_{1}, y_{1}\right) \approx\left(x_{2}, y_{2}\right)$ ] if $x_{1} y_{2}=x_{2} y_{1}$. The commutativity and cancellation properties are used to show that the relation $\approx$ is an equivalence relation (reflexive, symmetric, and transitive). The elements of the group are classes of equivalent pairs; the class of pairs equivalent to $(x, y)$ is denoted $\overline{(x, y)}$. The binary operation on such classes is defined componentwise: $\overline{\left(x_{1}, y_{1}\right)} \overline{\left(x_{2}, y_{2}\right)}=\overline{\left(x_{1} x_{2}, y_{1} y_{2}\right)}$. The componentwise operation can be shown easily (using commutativity and cancellation) to yield a result that depends only on the two classes being combined, not on the particular representatives of those classes chosen to compute the combination. Obviously the operation is associative and commutative. The class of "0-differences" (or "unit-ratios") $\overline{(x, x)}$ is an identity, since $(x y, x z) \approx(y, z)$; and $(\overline{y, x})$ is the inverse of $\overline{(x, y)}$. The original semigroup $X$ can be identified as part of this group by identifying $x$ with $(\bar{x} x, \bar{x})$. Obviously, $\overline{(x x, x)}=\overline{(y y, y)}$ if and only if $x=y$, and also, $\overline{(x x, \bar{x})}(\overline{y y, y)}=\overline{(x y x y, x y)}$, so the binary operation in $X$ goes over into the binary operation in the "group of differences." The term "group of differences" will be used below to describe this construction, and the group constructed from $X$ will be denoted $\bar{X}$.

The importance of the group of differences lies in the following considerations. If $A, B, C$ are stimulus elements, and $a$ is a transformation in a semigroup of transformations, $\mathscr{G}$, with

$$
A^{T} \equiv B^{S}, \quad A^{\alpha T} \equiv C^{S},
$$

then it may not be reasonable to assume that there will always be $b$ in $\mathscr{G}$ such that $B^{b}=C$. However, it may be quite reasonable to assume that there will exist $b, c$ in $\mathscr{G}$ such that $B^{b}=C^{c}$. (See the following paragraph.) Thus, to a change in appearance $a$, under context $T$, there corresponds a difference in appearance changes, $(b, c)$, under context $S$. Context changes (from $S$ to $T$ ) can thus be represented by a transformation in the group of differences $\overline{\mathscr{G}}$ (a transformation of transformation-differences, as it were).

Let $X$ be a semigroup of transformations acting in a set $K . X$ is said to be complete with respect to $K$ if for every $k, \ell$ in $K$, there exist $x, y$ in $X$ such that $k^{x}=\ell^{y}$.

If $X$ is a commutative semigroup of one-to-one transformations, complete with respect to $K$, then it is possible to define an induced group structure over $K$. More precisely, it is possible to define an equivalence relation $\approx$ on pairs of elements of $K$, and a binary composition + on the set $\bar{K}$ of equivalence classes, such that $\bar{K}$ is a commutative group which is isomorphic to $\bar{X}$.

The construction is particularly simple in the case where there is a single element $k_{0}$ in $K$ such that for any $k$ in $K$, there exists some $x$ in $X$ such that $k_{0}{ }^{x}=k$. (In the example considered in Sec. 1 , where $K$ is the set of spectral energy distributions, and the transformations are additions of any fixed distributions, take $k_{0}$ as the distribution with 0 encrgy density at cvery wavelength, i.e., the "blank" stimulus element; 
then $k=k_{0}+k$ for any $k$.) Define a function $\varphi$ by $\varphi(x)-k_{0}{ }^{x}$. It is easy to show that $\varphi$ is a one-to-one mapping of $X$ onto $K$. Thus, one obtains a commutative cancellation semigroup structure on $K$, via the binary operation $k_{0}^{x}+k_{0}{ }^{y}=k_{0}^{x y}$. In this case, the group structurc $\bar{K}$ is exactly the group of differences of the semigroup $K$.

When no element $k_{0}$ exists such that every $k$ can be written in the form $k_{0}{ }^{x}$, one can use completeness of $X$ with respect to $K$ to associate pairs $(k, \ell)$ with the elements of $\bar{X}:$ if $k^{x}=\ell$, associate $(k, \ell)$ with $\overline{(y, x)}$. By completeness, such $x$ and $y$ can always be found, and they determine an unique equivalence class, i.e., an unique element of $\bar{X}$, as the following argument shows. Suppose that $k^{x}=\ell{ }^{y}$. Then for any $x^{\prime}, y^{\prime}$,

$$
\begin{aligned}
k^{x^{\prime}}=\ell^{y^{\prime}} & \text { if and only if } k^{x^{\prime} x}=\ell^{y^{\prime} x}, \\
& \text { if and only if } \ell^{x^{\prime} y}=\ell^{x y^{\prime}}
\end{aligned}
$$

(the second step follows from the first by several uses of commutativity and by substitution of $\ell^{y}$ for $k^{x}$ on the left). It turns out that if $\ell^{x^{\prime} y}=\ell^{x y^{\prime}}$, for just one $\ell$, then $x^{\prime} y=x y^{\prime}$; so it follows that if $k^{x}=\ell^{y}$, then $k^{x^{\prime}}=\ell y^{\prime}$ if and only if $(x, y) \approx\left(x^{\prime}, y^{\prime}\right)$; so that any pair $(k, \ell)$ is associated with all the members and only the members of one equivalence class $\overline{(y, x)}$. The proof that $x^{\prime} y=x y^{\prime}$ is based on the properties of completeness, commutativity, and one-to-oneness of $X$; this fact is worth stating separately as a lemma:

LEMMA. Let $X$ be a commutative semigroup of one-to-one transformations, complete with respect to $K$. If for some $k$ in $K, k^{x}=k^{y}$, then $x=y$.

Proof. For every $\ell$ in $K$, there exists $z, w$ such that $k^{z}=\ell^{w}$. Then $\ell^{x w}=\ell^{w x}=$ $k^{z x}=k^{x z}=k^{y z}=k^{z y}=\ell^{u y}=\ell^{y w}$. Since $w$ is one-to-one, $\ell^{x}=\ell^{y}$. Thus, $x=y$.

Q.E.D.

Using the above, one can define $(k, \ell) \approx\left(k^{\prime}, \ell^{\prime}\right)$ if they are associated with the same element of $\bar{X}$; this is obviously an equivalence relation. Let $(\overline{k, \ell})$ be the equivalence class containing $(k, \ell)$ and let $\bar{K}$ be the set of equivalence classes. For any $k$ in $K$ the pair $\left(k^{x}, k^{y}\right)$ is associated with $(\overline{x, y)}$; thus, one can write all elements of $\bar{K}$ in the form $\overline{\left(k^{x}, k^{y}\right)}$ for some fixed $k$, and the componentwise binary operation in $\bar{X}$ can be transferred to $\bar{K}$ :

$$
\overline{\left(k^{x}, k^{y}\right)}+\overline{\left(k^{x^{\prime}}, k^{y^{\prime}}\right)}=\overline{\left(k^{x x^{\prime}}, k^{y y^{\prime}}\right)}
$$

The mapping associating elements of $\bar{K}$ with elements of $\bar{X}$ is a group isomorphism (one-to-one, onto, preserving binary operations).

In the case where $k_{0}$ exists such that every $k$ can be written as $k_{0}{ }^{x}$ for some $x$, every element $k$ in $K$ can be represented in the group of differences by $\left(\overline{\left.k_{0}^{x x}, k_{0}^{x}\right)}\right.$, or more simply, by $\left(\overline{\left.k_{0} x, k_{0}\right)}\right.$. In the general case, one simply chooses an arbitrary reference point, labelling it $k_{0}$, and associates the element $k$ with $\overline{\left(k, k_{0}\right)}$, its "difference" from 
$k_{0}$. The following formula, which can easily be proved, will be useful later: for any $k_{0}, k, \ell$,

$$
\overline{(\ell, k)}+\overline{\left(k, k_{0}\right)}=\overline{\left(\ell, k_{0}\right)} .
$$

The above construction of induced group structure shows that the presence of structure in a semigroup of transformations can be used to define structure in a set of stimulus elements. In Sec. 5 it is used to represent context change by a transformation in spaces of stimulus elements.

\section{DEFINITIONS AND BASIC ASSUMPTIONS}

Four types of objects must be considered in cross-context matching:

a set $\mathscr{C}$ of stimulus elements, $A, B$, etc.

a set $\mathscr{S}$ of contexts, $S, T$, etc.

a set $\mathscr{G}$ of transformations of $O d, a, b$, etc.

a set $\mathscr{H}$ of transformations of $\mathscr{S}, s, t$, etc.

The basic structure on $\mathscr{C}$ and $\mathscr{S}$ is a cross-context matching relation $\equiv$, on the

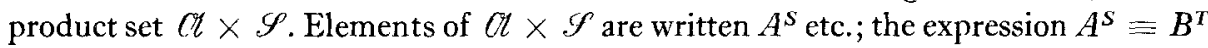
means "stimulus element $A$, in context $S$, matches stimulus element $B$, in context $T$ (with respect to the specified subjective dimensions)." The results of transforming $A$ by $a$, or $S$ by $s$, will be written $A^{a}$ and $S^{s}$ respectively. Parentheses will be omitted where possible; for instance, the notation $A^{a S}$ will be used instead of the more explicit $\left(A^{a}\right)^{S}$.

The relation $\equiv$ induces relations on $C$, one for each context $S$ : the relation $=s$ will be defined by $A={ }_{s} B$ if $A^{S} \equiv B^{S}$. In the case of color stimuli, two stimuli that match in the same context are called metamers, and, over an important range of conditions, metameric matches are independent of context, i.e., the relations $={ }_{s}$ are the same for all $S$. (See Brindley, 1960, for discussion of this point.)

The first assumption concerns the relation $\equiv$.

Assumption 1. The relation $\equiv$ on $\mathscr{O} \times \mathscr{S}$ is an equivalence relation (reflexive, symmetric, and transitive).

This implies, of course, that all the relations $=s$ are also equivalence relations. Empirical matching data, of course, cannot be expected to satisfy transitivity, unless extreme precautions are taken to ensure that obtained matches are exact, rather than due to failures of discrimination. This can be partially achieved by rejecting a match for a given pait of stimuli whenever a third stimulus can be found that "matches" one of the pair but not the other. By this sort of refinement one can get as good an approximation to transitivity as needed. Perfect transitivity is an idealization, which could be realized only if a given match were tested as indicated against all possible third stimuli, before acceptance (see Luce, 1956; Zeeman, 1962). 
Reflexivity and symmetry of a matching relation can also fail empirically, because of time-order or space-order errors, or the like, but they can usually be restored by appropriate operational definitions of "matching." For example, if successive comparisons are used, define two stimuli as "matching" if, when both are presented following some third stimulus (i.e., on separate trials), they both match that third stimulus on the relevant dimensions. If stimuli are compared simultaneously, in different eyes or in different parts of the visual field, define two stimuli as "matching" if they both match the same third stimulus in the other eye or other region of the visual field.

Assumptron 2. (i) $\mathscr{G}$ is a commutative semigroup of one-to-one transformations of $M$, which is complete with respect to $O$ (see Sec. 2 for definition).

(ii) For any $b$ in $\mathscr{G}$, and any $A, B$ in $\mathscr{U}, S$ in $\mathscr{F}, A={ }_{S} B$ if and only if $A^{b}={ }_{S} B^{b}$.

The meaning of part (i) is clear from Sec. 2. Part (ii) says that to any transformation $b$ in $\mathscr{G}$, there corresponds a one-to-one transformation, denoted $b_{S}$, of the set of equivalence classes in $a$ with respect to the equivalence relation $=s$. The class of all stimulus elements that are $=_{s}$ to $A$ will be denoted $A_{S}$, and the set of all such equivalence classes is denoted $a_{s}$. The transformation $b_{S}$ is defined by

$$
A_{S}^{b}=\left(A^{b}\right)_{S} .
$$

Part (ii) of Assumption 2 simply means that the transformation $h_{S}$ is well defined [since if $A_{S}=B_{S}$, then $A={ }_{s} B$, so $A^{b}={ }_{S} B^{b}$, or $\left(A^{b}\right)_{S}=\left(B^{b}\right)_{S}$ ], and one-to-one (since the steps in the brackets can all be reversed). Thus, for each context $S$, one has a commutative semigroup $\mathscr{G}_{S}$ of one-to-one transformations of $\mathscr{C}_{S}$, complete with respect to $C_{S}$. The only point to note is that for any $a_{S}, b_{S}$, the transformation $a_{S} b_{S}$ is in $\mathscr{G}_{S}$, since it is exactly equal to $(a b)_{S}$. Commutativity and completeness of $\mathscr{G}_{S}$ are obvious.

It is hard to discuss the general plausibility of Assumption 2 without specifying the nature of the stimulus domain and the type of transformations in $\mathscr{G}$. In one case, however, Assumption 2 is satisfied. If $C l$ consists of the spectral energy distribution functions, and the transformations in $\mathscr{G}$ each involve addition of some fixed distribution, then part (i) of Assumption 2 follows on the basis of consideration of physics alone. Part (ii) is a special case of Grassman's Third Law of color mixture (see Judd, 1951).

Definition $1 . G$ is said to be context-invariant with respect to $S, T$ provided that for any $a, A_{1}, B_{1}, C_{1}, b_{1}, c_{1}, A_{2}, B_{2}, C_{2}, b_{2}$, and $c_{2}$, if

$$
\begin{aligned}
A_{1}^{T} \equiv B_{1}{ }^{S}, & A_{2}^{T} \equiv B_{2}{ }^{S}, \\
A_{1}^{a T} \equiv C_{1}{ }^{S}, & A_{2}^{a T} \equiv C_{2}{ }^{S}, \\
B_{1}^{b_{1}}=C_{1}^{c_{1}}, & B_{2}^{b_{2}}=C_{2}^{c_{2}},
\end{aligned}
$$


then $\left(b_{1} c_{2}\right)_{S}=\left(b_{2} c_{1}\right)_{S} . \mathscr{G}$ is said to be context-inasariant with respect to $\mathscr{S}$ if it is context-invariant with respect to $S, T$ for each $S$ and $T$ in $\mathscr{S}$.

Context invariance with respect to $S, T$ means that if transformation $a$, under context $T$, produces an appearance change that is matched by the difference between stimulus elements $C$ and $B$ in context $S$, this change can be represented in context $S$ by a transformation difference $\left(b_{S}, c_{S}\right)$, whose equivalence class in the group of differences $\overline{\mathscr{G}}_{S}$ depends only on $a$, not on the choice of $A_{1}$ or $A_{2}$. This concept was discussed in detail in Sec. 1.

\section{Assumption. 3. $\mathscr{G}$ is context-invariant with respect to $\mathscr{S}$}

Assumption 3 is the major substantive assumption of the theory. It has not often been tested, even for addition-transformations of spectral energy distributions, although for this special case, the data of Burnham, Evans, and Newhall (1957), described in Sec. 10, provide support. As mentioned in the first section, one conceivable strategy in studying context effects is to look for a semigroup of context-invariant transformations.

Clearly, context-invariance suggests representation of the change from context $S$ to context $T$ by a mapping which takes the transformation $a_{T}$, occurring in Def. 1, into the difference $\overline{\left(b_{S}, c_{S}\right)}$ in $\overline{\mathscr{G}}_{S}$. To do this, it must be possible to find some $A$ such that the appearances of both $A^{T}$ and $A^{a T}$ can be matched by some stimulus elements under context $S$. This requires some existence assumption; the exact assumption needed is formulated in the next definition.

Definition 2. Let $S_{1}, \ldots, S_{n}$ be contexts in $\mathscr{S}$. They are said to overlap if for any $b, c$ in $\mathscr{G}$ and for any index $j, j=1, \ldots, n$, there exist elements $A, A_{1}, \ldots, A_{n}$, $B_{1}, \ldots, B_{n}, C_{1}, \ldots, C_{n}$ in $O$ such that

$$
\begin{gathered}
A^{S_{j}} \equiv A_{1}^{S_{1}} \equiv \cdots \equiv A_{n}^{S_{n}} \\
A^{b S_{j}} \equiv B_{1}^{S_{i}} \equiv \cdots \equiv B_{n}^{S_{n}} \\
A^{c S_{j}} \equiv C_{1}^{S_{1}} \equiv \cdots \equiv C_{n}^{S_{n}} .
\end{gathered}
$$

(Obviously one can take $A_{j}=A, B_{j}=A^{b}, C_{j}=A^{c}$.)

In other words, $S_{1}, \ldots, S_{n}$ overlap if they contain sufficiently many subjective appearances in common, so that for any two transformations $b, c$, one can find $A$ such that it, and its two transforms $A^{b}$ and $A^{c}$, appearing in context $S_{j}$, can be matched in appearance in the other $n-1$ contexts. The definition of course is symmetric, since the property must hold for every $j$. 
It is not reasonable to assume in general that sets of $n$ contexts, or even sets of 2 contexts overlap. What will be assumed is that contexts that are sufficiently similar do overlap. This assumption will be tacit in Secs. 4-6; the hypothesis that $S, T$ overlap, or that $S, T, U$ overlap, will occur in the statement of definitions and theorems, and the tacit assumption is that this hypothesis will sometimes be satisfied, so that the definitions and theorems are not vacuous. In Sec. 7, rigorous definitions of "sufficiently similar" will be available (in terms of an assumed topology on contexts) and a formal assumption about overlap (Assumption 6) will be introduced; at the same time, the theory will be extended to deal with the case of nonoverlapping contexts.

Assumption 4. If $A_{1}{ }^{T} \equiv B_{1}{ }^{S}$ and $A_{2}{ }^{T} \equiv B_{2}{ }^{S}$, then there exist $a_{1}, a_{2}$ in $\mathscr{G}$ and $B$ in Ot such that

$$
A_{1}^{a_{1}}=A_{2}^{a_{2}} \quad \text { and } \quad A_{1}^{a_{1} T} \equiv A_{2}^{a_{2} T} \equiv \equiv B^{S} .
$$

According to this assumption, if $A_{1}$ and $A_{2}$ yield appearances in context $T$ which can be matched in context $S$, then the transformations $a_{1}$ and $a_{2}$ can be chosen so as to move $A_{1}$ and $A_{2}$ to an appearance "between" that of $A_{1}$ and $A_{2}$, which can also be matched in context $S$.

The above definitions and assumptions concern only $\mathscr{O}, \mathscr{P}$, and $\mathscr{G}$; the set $\mathscr{H}$ of transformations of $\mathscr{P}$ was not mentioned. Its structure will be considered in Sec. 6 , where Assumption 5, a second major substantive assumption, will be introduced.

\section{THE REPRESENTATION OF CONTEXT CHANGES BY ISOMORPHISMS OF TRANSFORMATION GROUPS}

Definition 3. Suppose that contexts $S, T$ overlap (Def. 2). For any $a$ in $\mathscr{G}$, find $A, B, C$ in $C l$ such that

$$
\begin{aligned}
A^{T} & \equiv B^{S}, \\
A^{a T} & \equiv C^{S} .
\end{aligned}
$$

By completeness [Assumption 2, (i)], find $b, c$ in $\mathscr{G}$ such that $B^{b}=C^{c}$. By contextinvariance (Assumption 3), the equivalence class $\overline{\left(b_{S}, c_{S}\right)}$ in $\overline{\mathscr{G}}_{S}$ determined by $b, c$ is independent of the choice of $A, B$, and $C$; clearly it is also dependent only on the transformation $a_{T}$ in $\mathscr{G}_{T}$ determined by $a$, i.e., if $a_{1 T}=a_{2 T}$, then even if $a_{1} \neq a_{2}$, the same $B, C$ can be used, so the same $\left.\overline{\left(b_{S}, c_{S}\right.}\right)$ will necessarily result. Thus, the above construction defines a function, denoted $f_{S, T}$, mapping $\mathscr{G}_{T}$ into $\overline{\mathscr{G}}_{S}$, with $\int_{S, T}\left(a_{T}\right)=\overline{\left(b_{S}, c_{S}\right)}$.

The function $f_{S, T}$, mapping the semigroup $\mathscr{G}_{T}$ into the group $\overline{\mathscr{G}}_{S}$, describes the effect of changing from context $S$ to context $T$, for two overlapping contexts $S, T$. 
The surprising fact is that the function $f_{S, T}$ defined in this way is necessarily multiplicative, as shown in Theorem 1.

Lemma. Let $A_{1}, \ldots, A_{n}$ be arbitrary elements of $O$. There exist transformations $a_{1}, \ldots, a_{n}$ in $\mathscr{G}$ such that

$$
A_{1}^{a_{1}}=\cdots=A_{n}^{a_{n}}
$$

Proof. For each $i, i-2, \ldots, n$, one can find $b_{i}, c_{i}$ such that

$$
A_{1}^{b_{i}}=A_{i}^{c_{i}}
$$

[completeness, Assumption 2, (i)]. Now define $a_{1}$ to be the product of $b_{2}$ through $b_{n}$, while for $i=2, \ldots, n$, define $a_{i}$ to be the product of $c_{i}$ and all the $b$ 's except $b_{i}$. Obviously for $i=2, \ldots, n$

$$
A_{1}^{a_{1}}=A_{i}^{a_{i}}
$$

Theorem 1. Suppose $S, T$ overlap. Then for any $a_{1}, a_{2}$,

$$
f_{S . T}\left(a_{1 T} a_{2 T}\right)=f_{S . T}\left(a_{1 T}\right) f_{S . T}\left(a_{2 T}\right) .
$$

Proof. By the definition of overlap (Def. 2) one can choose $A, B, C_{1}$, and $C_{2}$ such that

$$
\begin{aligned}
A^{T} & \equiv B^{S}, \\
A^{a_{1} T} & \equiv C_{1}{ }^{S}, \\
A^{a_{1} \alpha_{2} T} & \equiv C_{2}{ }^{s} .
\end{aligned}
$$

By the previous lemma, there exist $b, c_{1}, c_{2}$ such that

$$
B^{b}=C_{1}^{c_{1}}=C_{2}^{c_{2}} .
$$

By Def. 3,

$$
\begin{aligned}
f_{S, T}\left(a_{1 T}\right) & =\overline{\left(b_{S}, c_{1 S}\right)}, \\
f_{S, T}\left(a_{2 T}\right) & =\overline{\left(c_{1 S}, c_{2 S}\right)}, \\
f_{S, T}\left(a_{1 T} a_{2 T}\right) & =\overline{\left(b_{S}, c_{2 S}\right)} .
\end{aligned}
$$

(Note that use has been made of the freedom provided by context-invariance: $f_{S, T}\left(a_{2 T}\right)$ is computed using $A^{a_{1}}$ as the starting point while the other two values of 
$f_{S, T}$ are computed starting with $A$.) By the definition of multiplication in the group of differences $\overline{\mathscr{G}}_{S}$,

$$
\begin{aligned}
\overline{\left(b_{S}, c_{1 S}\right)} \overline{\left(c_{1 S}, c_{2 S}\right)} & =\overline{\left(b_{S} c_{1 S}, c_{2 S} c_{1 S}\right)} \\
& =\overline{\left(b_{S}, c_{2 S}\right)} .
\end{aligned}
$$

The multiplicative property exhibited in Theorem 1 allows the extension of the definition of $f_{S, T}$ to a mapping of all of $\overline{\mathscr{G}}_{T}$ into $\overline{\mathscr{G}}_{S}$. This is done in Def, 4.

Definition 4. Let $S, T$ overlap. For $\overline{\left(a_{1 T}, a_{2 T}\right)}$ in $\overline{\mathscr{G}}_{T}$, define $f_{S, T} \overline{\left(a_{1 T}, a_{2 T}\right)}=$ $f_{S, T}\left(a_{1 T}\right)\left[f_{S, T}\left(a_{2 T}\right)\right]^{-1}$ [where $(g)^{-1}$ denotes the inverse of an element $g$ of $\overline{\mathscr{G}}_{S}$ ].

Theorem 2. If $S, T$ overlap, then $f_{S, T}$ is a well defined, one-to-one mapping of $\overline{\mathscr{G}}_{T}$ onto $\overline{\mathscr{G}}_{S}$, satisfying

$$
f_{S, T}\left(g_{1} g_{2}\right)=f_{S, T}\left(g_{1}\right) f_{S, T}\left(g_{2}\right)
$$

(where $g_{1}, g_{2}$ denote two arbitrary elements of $\overline{\mathscr{G}}_{T}$ ). Thus $f_{S, T}$ is an isomorphism of $\overline{\mathscr{G}}_{T}$ onto $\overline{\mathscr{G}}_{S}$.

Proof. First, one can show that $f_{S, T}$ is well defined, by means of the following series of implications: If

$$
\begin{aligned}
\overline{\left(a_{1 T}, b_{1 T}\right)}= & \overline{\left(a_{2 T}, b_{2 T}\right)}, \text { then } \quad\left(a_{1} b_{2}\right)_{T}=\left(a_{2} b_{1}\right)_{T}, \\
& \text { so } f_{S, T}\left(a_{1 T} b_{2 T}\right)=f_{S, T}\left(a_{2 T} b_{1 T}\right), \\
& \text { so } f_{S, T}\left(a_{1 T}\right) f_{S, T}\left(b_{2 T}\right)=f_{S, T}\left(a_{2 T}\right) f_{S, T}\left(b_{1 T}\right), \\
& \text { so } f_{S, T}\left(a_{1 T}\right)\left[f_{S, T}\left(b_{1 T}\right)\right]^{-1}=f_{S, T}\left(a_{2 T}\right)\left[f_{S, T}\left(b_{2 T}\right)\right]^{-1}, \\
& \text { so } f_{S, T} \overline{\left(a_{1 T}, b_{1 T}\right)}=f_{S, T} \overline{\left(a_{2 T}, b_{2 T}\right)} .
\end{aligned}
$$

Showing that $f_{S, T}$ is one-to-one requires that this chain of implications be reversed. This is trivial, except for the second implication, whose converse asserts that $f_{S, T}$ is one-to-one as a mapping of $\mathscr{G}_{T}$. The latter fact can be established as follows: Suppose that $f_{S . T}\left(a_{1 T}\right)=f_{S . T}\left(a_{2 T}\right)$. It must be shown that $a_{1 T}=a_{2 T}$. From the definition of overlap, there exist $A, B, C_{1}$, and $C_{2}$ such that

$$
\begin{aligned}
A^{T} & \equiv B^{S}, \\
A^{a_{1} T} & \equiv C_{1}{ }^{S}, \\
A^{a_{2} T} & \equiv C_{2}{ }^{S} .
\end{aligned}
$$


By the lemma of this section, $b, c_{1}, c_{2}$ can be chosen such that

$$
B^{b}=C_{1}^{c_{1}}=C_{2}^{c_{2}}
$$

Since $f_{S, T}\left(a_{1 T}\right)=f_{S, T}\left(a_{2 T}\right)$, one has $\overline{\left(b_{S}, c_{1 S}\right)}=\overline{\left(b_{S}, \overline{c_{2 S}}\right)}$, or $\left(b c_{1}\right)_{S}=\left(b c_{2}\right)_{S}$. Now we know that

$$
C_{1}^{b c_{1}}=C_{2}^{b c_{2}}
$$

so that

$$
C_{1}^{b c_{1} S} \equiv C_{2}^{b c_{2} S} \equiv C_{2}^{b c_{1} S} .
$$

By part (ii) of Assumption 2, it follows that $C_{1} s \equiv C_{2} s$. But, by the transitivity of $\equiv$, it follows that

$$
A^{a_{1} T} \equiv A^{a_{2} T} .
$$

In other words, the transformations $a_{1 T}$ and $a_{2 T}$ have the same effect on the element $A_{T}$ of $C_{T}$. Since the semigroup $\mathscr{G}_{T}$ is a commutative semigroup of one-to-one transformations of ${C Z_{T}}_{T}$, complete with respect to $\mathscr{C}_{T}$, the lemma of Sec. 2 applies, yielding the result $a_{1 T}=a_{2 T}$, as was to be proved. Thus, $f_{S, T}$ is one-lo-one.

The proof that $f_{S, T}$ is multiplicative on $\overline{\mathscr{G}}_{T}$ is straightforward from Def. 4 and Theorem 1. Finally, to show that $f_{S, T}$ is onto, one must produce, for any element of $\overline{\mathscr{G}}_{S}$, something in $\overline{\mathscr{G}}_{T}$ which maps onto it. The correct choice in $\overline{\mathscr{G}}_{T}$ turns out to be $f_{T, S}$ of the given element in $\overline{\mathscr{G}}_{S} ; f_{S, T}$ and $f_{T, S}$ turn out to be inverse mappings of one another. This last fact is a corollary of Theorem 3 below.

Q.E.D.

It should be noted that, up to this point, no use has been made of Assumption 4 . It is used, however, in the next theorem, which shows that if one combines the isomorphisms that go from context $S$ to context $T$ and from context $T$ to context $U$, one gets the isomorphism from context $S$ to context $U$.

Theorem 3. Suppose that $S, T, U$ overlap. Then for any $\overline{\left(a_{1 U}, a_{2 U}\right)}$ in $\overline{\mathscr{G}}_{U}$,

$$
f_{S, T} f_{T, U}\left(\overline{\left.a_{1 U}, a_{2 U}\right)}=f_{S, U} \overline{\left(a_{1 U}, a_{2 U}\right)}\right. \text {. }
$$

Proof. It is simplest to begin by showing that the theorem holds for any element $a_{U}$ in $\mathscr{G}_{U}$. Let $a_{U}$ be arbitrary. Since $S, T, U$ overlap, there exist $A, B, C, B_{1}$, and $C_{1}$ such that

$$
\begin{aligned}
A^{U} & \equiv B^{T} \equiv C^{S}, \\
A^{a U} & \equiv B_{1}{ }^{T} \equiv C_{1}{ }^{S} .
\end{aligned}
$$


By Assumption 4, there exist $b, b_{1}$, and $D$ such that

$$
B^{b}=B_{1}^{b_{1}} \quad \text { and } \quad B^{b T} \equiv B_{1}^{b_{1} T}=D^{S} .
$$

By the lemma of this section, there exist $c, c_{1}$, and $d$ such that

$$
C^{c}=C_{1}^{c_{1}}=D^{d} .
$$

By Def. 3,

$$
\begin{aligned}
f_{T, U}\left(a_{U}\right) & \left.=\overline{\left(b_{T}, b_{1 T}\right.}\right), \\
f_{S, U}\left(a_{U}\right) & =\overline{\left(c_{S}, c_{1 S}\right)}, \\
f_{S, T}\left(b_{T}\right) & =\overline{\left(c_{S}, d_{S}\right)}, \\
f_{S, T}\left(b_{1 T}\right) & =\overline{\left(c_{1 S}, d_{S}\right)} .
\end{aligned}
$$

By Def. 4,

$$
\begin{aligned}
f_{S, T} f_{T, U}\left(a_{U}\right) & =f_{S, T}\left(b_{T}\right)\left[f_{S, T}\left(b_{1 T}\right)\right]^{-1} \\
& =\overline{\left(c_{S}, d_{S}\right)} \overline{\left(d_{S}, c_{1 S}\right)} \\
& =\overline{\left(c_{S}, c_{1 S}\right)} \\
& =f_{S, U}\left(a_{U}\right) .
\end{aligned}
$$

From this result, plus breakdown of $f_{T, U} \overline{\left(a_{1 U}, a_{2 U}\right)}$ by Def. 4 and the multiplicative property of $f_{S, T}$ from Theorem 3 , it is easy to verify the formula stated in the theorem.

Q.E.D.

CoROLlary. $f_{S, S}$ is the identity mapping of $\overline{\mathscr{G}}_{S}$ (it maps each element into itself). If $S, T$ overlap, then $f_{S, T}$ and $f_{T, S}$ are inverse to one another.

Pronf. It is fairly obvious that $f_{S, S}(g)=g$ for any element $g$ in $\overline{\mathscr{G}}_{S}$. This fact can be proved from the theorems above: since $S, S, S$ overlap,

$$
\begin{aligned}
f_{S, s}\left\{\left[f_{S, s}(g)\right]\left[(g)^{-1}\right]\right\} & -f_{S, s}\left[f_{S, s}(g)\right] f_{S, s}\left(g^{-1}\right) \\
& =f_{S, s}(g) f_{S, s}\left(g^{-1}\right) \\
& -f_{s, s}\left[(g)\left(g^{-1}\right)\right] .
\end{aligned}
$$

Since $f_{S, S}$ is one-to-one, the arguments on the left and right sides are equal, and cancelling $g{ }^{1}$ from both of them leaves $f_{s, s}(g)=g$. 
If $S, T$ overlap, then trivially, $S, T, S$ overlap and $T, S, T$ overlap, so for any $g$ in $\overline{\mathscr{G}}_{S}$,

$$
f_{S, T} f_{T, S}(g)=f_{S, S}(g)=g,
$$

and similarly, for $g$ in $\overline{\mathscr{G}}_{T}$,

$$
f_{T, S} f_{S, T}(g)=f_{T, T}(g)=g .
$$

The above corollary shows that $f_{S, T}$ is necessarily a mapping onto all of $\overline{\mathscr{G}}_{S}$, thereby completing the proof of Theorem 2.

It should be remarked that the notation and the results are considerably simpler if it is the case that for all $A, B, S$, and $T$,

$$
A^{S} \equiv B^{S} \quad \text { if and only if } \quad A^{T} \equiv B^{T} .
$$

As was noted above, this is possible, over a wide range of conditions, for color matching. When this property holds, the relations $=s$ and the sets $C_{S}$ of $={ }_{s}$-equivalence classes are the same for every $S$, and the semigroups $\mathscr{G}_{S}$ and the groups $\overline{\mathscr{G}}_{S}$ are all identical. Let all these identical groups be denoted by $\overline{\mathscr{G}}$. The mappings $f_{S, T}$ are automorphisms of $\overline{\mathscr{G}}$ onto itself, and one has a function

$$
(S, T) \rightarrow f_{S, T}
$$

sending pairs of overlapping contexts into elements of the group of automorphisms of $\overline{\mathscr{G}}$, with

$$
f_{S, T} f_{T, U}=f_{S, U} .
$$

Thus, in the search for consequences of structure, one ascends three levels of functional relations, considering transformations of stimulus elements, isomorphisms of transformation groups, and functions from pairs of contexts into the group of automorphisms of a transformation group. When group structure can be introduced into the set of pairs of contexts, this third step yields interesting results. This idea is carried out in Secs. 6-8 below.

The functions $f_{S, T}$ provide a reasonably good description of the effects on appearance of changing from context $S$ to context $T$. For example, if one knows $f_{S, T}$, one can predict (in terms of appearances under context $S$ ) some of the appearances under context $T$ from knowledge of other appearances under context $T$. For example, one can certainly predict the appearance (in terms of some standard context $S$ ) of $A^{a T}$, if one knows the appearance of $A^{T}$ and the transformation $f_{S, T}$. This predictive power from knowledge of $f_{S, T}$ will become even clearer in the next section. Granted the importance of $f_{S, T}$ as a description of appearances in context $T$ in terms of appearances in context $S$, it is significant that $f_{S, T}$ is not just any function, whose description requires a "graph" giving values for every possible element of $\overline{\mathscr{G}}_{T}$, but is an isomorphism of one commutative group onto another commutative group. Commutative 
groups are among the best-understood objects in mathematics. The exact advantage that can be taken of the fact that $f_{S, T}$ is a group isomorphism will depend somewhat on the particular structure of the group. For groups that are finitely generated, the situation is especially favorable, for all elements are products of elements in a certain finite set, and since isomorphisms preserve products, knowledge of $f_{S, T}$ for a finite number of elements of its domain allows prediction of its values for all elementsinstead of a graph, one has a finite number of "parameters." In the case of transformations of color stimuli, by adding a fixed spectral energy distribution, the group $\overline{\mathscr{G}}$ is a three-dimensional vector space, so knowledge of $f_{S, T}$ for just three elements in $\overline{\mathscr{G}}$ is sufficient to predict its values for all others; as will be pointed out in Secs. 5 and 8 , in this case, a complete description of appearances in context $T$, in terms of those in context $S$, requires estimation only of the 12 entries in a $3 \times 4$ matrix. (Of course, this depends on verifying that the addition-transformations yield a contextinvariant semigroup.)

\section{THE REPRESENTATION OF CONTEXT CHANGES BY AFFINE TRANSFORMATIONS OF APPFARANCE SPACE}

Much more natural than describing context changes in terms of isomorphisms of transformation groups is a description in terms of the appearances themselves: if $A^{T} \equiv B^{S}$, why not say that this pairing defines a function, $g_{S, T}$, such that $g_{S, T}\left(A_{T}\right)=B_{S}$ ? That is, $g_{S, T}$ maps the "appearance space" $O_{T}$ of $={ }_{T}$-equivalence classes into the space $\Pi_{S}$ of $={ }_{s}$-equivalence classes. There are two reasons for using the transformation semigroups instead. For one thing, they provide additional structure, giving the hope of predicting many appearances from matching only a few, as described at the end of the last section. For another, the function $g_{S, T}$ described above cannot be defined for all elements $A_{T}$, unless there is complete overlap between the appearances possible under context $T$ and those possible under context $S$. In the case of color stimuli, complete overlap is quite unlikely in general; an example was cited in Sec. 1.

Under the present assumptions (Sec. 3), and using the results of Sec. 4, both of the above objections can be circumvented, and functions $g_{S, T}$ can be defined which directly map appearances under one context at least into appearance differences under another context.

For any $S$, the construction of Sec. 2 can be applied to the complete commutative semigroup of one-to-one transformations $\mathscr{G}_{S}$ acting in $\mathscr{M}_{S}$, to obtain a group $\bar{O}_{S}$ whose elements arc cquivalence classes of appearance differences. This group is isomorphic to the group $\overline{\mathscr{G}}_{S}$. The pair $\left(A_{S}, B_{S}\right)$ is associated with the element $\overline{\left(b_{S}, a_{S}\right)}$ of $\overline{\mathscr{G}}_{S}$ if $A^{a S} \equiv B^{b S}$, and the equivalence class of all such pairs, $\overline{\left(A_{S}, B_{S}\right)}$, can be denoted $h_{S} \overline{\left(b_{S}, a_{S}\right)}$, where $h_{S}$ is the isomorphism mapping $\overline{\mathscr{G}}_{S}$ onto $\overline{\mathscr{V}}_{S}$. The inverse 
isomorphism is denoted $h_{s}^{-1}$. The element $h_{s} \overline{\left(b_{s}, a_{s}\right)}$ can be written $\overline{\left(C^{a}{ }_{s}, C^{b}\right)}$ for any $C$, and the binary operation in ${\widetilde{\sigma_{s}}}_{s}$ can thus be computed from the rule

$$
\left.\overline{\left(C^{a_{1}} s, C^{b_{1}} s\right)}+\overline{\left(C^{a_{2}} s, C^{b_{2}} s\right.}\right)=\overline{\left(C^{a_{1} a_{2} s}, C^{b_{1} b_{2}}\right)} \text {. }
$$

The isomorphism rule,

$$
h_{S}\left[\overline{\left(b_{1 S}, a_{1 S}\right)} \overline{\left(b_{2 S}, a_{2 S}\right)}\right]=h_{S} \overline{\left(b_{1 S}, a_{1 S}\right)}+h_{S} \overline{\left(b_{2 S}, a_{2 S}\right)}
$$

is obvious. (Note the use of juxtaposition for the binary operation in $\overline{\mathscr{G}}_{S}$ and of + for that in $\left.\bar{q}_{s}.\right)$

Let $\theta$ be some arbitrary element of $a$, chosen to act as a zero from which differences are measured. (It plays the role of $k_{0}$ in Sec. 2 ; if it can be chosen so that every $A$ in $O t$ can be written in the form $\theta^{a}$ for some $a$ in $\mathscr{G}$, this should be done.) Identify the element $A_{S}$ in $\mathscr{C}_{S}$ with the element $\overline{\left(A_{S}, \theta_{S}\right)}$ in $\overrightarrow{\mathscr{G}}_{S}$. Note that the sum, $\overline{\left(A_{S}, \theta_{S}\right)}+\overline{\left(B_{S}, \theta_{S}\right)}$ cannot necessarily be written in the form $\overline{\left(C_{S}, \theta_{S}\right)}$ for some $C$; in fact, this can be done if and only if $A_{S}-\theta^{a}{ }_{S}$ and $B_{S}=\theta^{h}{ }_{S}$ for somc $a, b$.

Suppose that whenever $A^{T} \equiv B^{S}$, one defines $g_{S, T}\left(A_{T}\right)=\overline{\left(B_{S}, \theta_{S}\right)}$. This is well defined for those $A_{T}$ that can be matched, for if $A^{T} \equiv B_{1}{ }^{S} \equiv B_{2}{ }^{S}$, then clearly $\overline{\left(B_{1} S, \theta_{S}\right)}=\overline{\left(B_{2 S}, \theta_{S}\right)}$. If $A^{T} \equiv A_{1}{ }^{S}$ and $B^{T} \equiv B_{1}{ }^{S}$, then one has the formula

$$
g_{S, T}\left(B_{T}\right)=g_{S, T}\left(A_{T}\right)+\overline{\left(B_{1 S}, A_{1 S}\right)}
$$

(see Sec. 2 or the remarks in the preceding paragraphs). Now the important point is that in this case, $\overline{\left(B_{1 S}, A_{1 S}\right)}$ can be computed in terms of $\left(B_{T}, A_{T}\right)$ and the function $f_{S, T}$ from Sec. 4. This is shown in the next theorem.

Theorem 4. Let $A^{T} \equiv A_{1}{ }^{S}$ and $B^{T} \equiv B_{1}{ }^{S}$, where $S, T$ overlap.

Then

$$
\overline{\left(B_{1 S}, A_{1 S}\right)}=h_{S} f_{S, T} h_{T}^{-1} \overline{\left(B_{T}, A_{T}\right)} .
$$

Proof. By Assumption 4, it is possible to choose $a, b$, and $C$ such that

$$
A^{a T} \equiv B^{b T} \equiv C^{S} .
$$

By the lemma of Sec. 4 , it is possible to choose $a_{1}, b_{1}$, and $c$ such that

Now

$$
A_{1}^{a_{1}}=B_{1}^{b_{1}}=C^{c} .
$$

$$
\begin{aligned}
h_{T}^{-1} \overline{\left(B_{T}, A_{T}\right)} & =\overline{\left(a_{T}, b_{T}\right)} \\
f_{S . T} \overline{\left(a_{T}, b_{T}\right)} & =f_{S . T}\left(a_{T}\right)\left[f_{S, T}\left(b_{T}\right)\right]^{-1} \\
& =\overline{\left(a_{1 S}, c_{S}\right)} \overline{\left(c_{S}, b_{1 S}\right)} \\
& =\overline{\left(a_{1 S}, b_{1 S}\right)}
\end{aligned}
$$


and

$$
h_{S} \overline{\left(a_{1 S}, b_{1 S}\right)}=\overline{\left(B_{1 s}, A_{1 s}\right)} .
$$

In consequence of Theorem 4 and of the formula preceding it, the following cquation holds:

$$
g_{S, T}\left(B_{T}\right)=g_{S, T}\left(A_{T}\right)+h_{S} f_{S, T} h_{T}^{-1} \overline{\left(B_{T}, A_{T}\right)} .
$$

Equation 1 holds whenever $A_{T}$ and $B_{T}$ can both be matched within $C_{S}$. However, the second term on the right hand side is defined for any $A_{T}$ and $B_{T}$, provided $S, T$ overlap, and in that case, $g_{S . T}$ is also defined for at least some $A_{T}$; therefore, Eq. 1 can be used to define $g_{S, T}\left(B_{T}\right)$ for elements $B_{T}$ in $C_{T}$ whose appearance cannot be matched in $C_{S}$. In these cases, of course, the value of $g_{S, T}\left(B_{T}\right)$ is an element of $\bar{C}_{S}$, i.e., an appearance difference. Formally, one can state the following definition:

Definition 5. Suppose $S, T$ overlap. Choose $C$ and $C_{1}$ arbitrarily such that $C^{T} \equiv C_{1}{ }^{S}$. For all $B$, let $g_{S, T}\left(B_{T}\right)$ be defined by

$$
g_{S, T}\left(B_{T}\right)=\overline{\left(C_{1 S}, \theta_{S}\right)}+h_{S} f_{S, T} h_{T}^{-1} \overline{\left(B_{T}\right.}, \overline{\left.C_{T}\right)} .
$$

Theorem 5. Suppose $S, T$ overlap. Let $g_{S, T}$ be defined by Def. 5. Then

(i) Equation 1 is valid for all $A, B$;

(ii) the values of $g_{S, T}$ do not depend on the choice of $C$ in Def. 5;

(iii) if $B^{T} \equiv B_{1}{ }^{S}$, then $g_{S, T}\left(B_{T}\right)=\overline{\left(B_{1 S}, \theta_{S}\right)}$.

Proof. To prove (i), note that for any $A, B$,

$$
\begin{aligned}
g_{S, T}\left(A_{T}\right)+h_{S} f_{S, T} h_{T}^{-1} \overline{\left(B_{T}, A_{T}\right)} \\
=\overline{\left(C_{1 S}, \theta_{S}\right)}+h_{S} f_{S, T} h_{T}^{-1} \overline{\left(A_{T}, C_{T}\right)}+h_{S} f_{S, T} h_{T}^{-1} \overline{\left(B_{T}, A_{T}\right)} \\
=\overline{\left(C_{1 S}, \theta_{S}\right)}+h_{S} f_{S, T} h_{T}^{-1} \overline{\left[\left(A_{T}, C_{T}\right)\right.}+\overline{\left.\left(B_{T}, A_{T}\right)\right]} \\
=\overline{\left(C_{1 S}, \theta_{S}\right)}+h_{S} f_{S, T} h_{T}^{-1} \overline{\left(B_{T}, C_{T}\right)} \\
=g_{S, T}\left(B_{T}\right) .
\end{aligned}
$$

The second step in the above sequence required the use of the facts that $h_{T}^{-1}, f_{S, T}$, and $h_{S}$ are all group isomorphisms, so that the + sign can be transferred from outside the functions to inside the brackets. Statement (iii) follows immediately from Theorem 4, since $\overline{\left(C_{1 S}, \theta_{S}\right)}+\overline{\left(B_{1 S}, C_{1 S}\right)}=\overline{\left(B_{1 S}, \theta_{S}\right)}$. Finally, statement (ii) follows from (i) and (iii) since if $g_{S, T}\left(B_{T}\right)$ were defined using $A$ instead of $C$, where $A^{T} \equiv A_{1}{ }^{S}$, then the defining expression would be exactly the right side of Eq. 1, which is the value of $y_{S, T}\left(B_{T}\right)$ defined in terms of $C$, according to (i).

Q.E.D. 
Definition 5 and Theorem 5 show that the second difficulty mentioned at the beginning of this section can be overcome: $g_{S, T}$ can be defined, at least as an appearance difference, for all elements in the appearance space $C_{T}$. To deal with the first difficulty, lack of structure on $C_{T}$, one must seek to define $g_{S, T}$ in a reasonable way on the group ${\overrightarrow{\sigma_{T}}}_{T}$. One may start such a definition by identifying $g_{S, T} \overline{\left(A_{T}, \theta_{T}\right)}$ with $g_{S, T}\left(A_{T}\right)$. The question is, can one write a general definition of $g_{S, T}\left(\overline{\left.A_{T}, B_{T}\right)}\right.$ in terms of $g_{S, T}\left(A_{T}\right)$ and $g_{S, T}\left(B_{T}\right)$ ?

Note that for any $a, b$,

$$
\overline{\left(\theta^{a b}{ }_{T}, \theta^{a}{ }_{T}\right)}=\overline{\left(\theta^{b}{ }_{T}, \theta_{T}\right)}
$$

Thus,

$$
\begin{aligned}
g_{S, T} \overline{\left(\theta^{a b}{ }_{T}, \theta^{a} T\right)} & =g_{S, T}\left(\theta_{T}^{b}\right) \\
& =g_{S, T}\left(\theta_{T}\right)+h_{S} f_{S, T} h_{T}^{-1} \overline{\left(\theta^{b}{ }_{T}, \theta_{T}\right)} \\
& =g_{S, T}\left(\theta_{T}\right)+h_{S} f_{S, T} h_{T}^{-1} \overline{\left(\theta^{a b}{ }_{T}, \theta_{T}\right)}-h_{S} f_{S, T} h_{T}^{-1} \overline{\left(\theta^{a}, \theta_{T}\right)} \\
& =g_{S, T}\left(\theta_{T}\right)+g_{S, T}\left(\theta^{a b}{ }_{T}\right)-g_{S, T}\left(\theta^{a}{ }_{T}\right) .
\end{aligned}
$$

The second step of the sequence is justified by the isomorphism properties of $h_{S} f_{S, T} h_{T}^{-1}$ and by the fact that

$$
\overline{\left(\theta^{b}{ }_{T}, \theta_{T}\right)}=\overline{\left(\theta^{a b}{ }_{T}, \theta_{T}\right)}-\overline{\left(\theta^{a}{ }_{T}, \theta_{T}\right)} .
$$

The above calculation of $g_{S, T} \overline{\left(\theta^{a b} T, \theta_{T}^{a}\right)}$ suggests the following general definition:

Definition 6. Let $S, T$ overlap and let $g_{S, T}$ be defined on $C_{T}$ as in Def. 5. For all $A, B$, define

$$
g_{S, T} \overline{\left(A_{T}, B_{T}\right)}=g_{S, T}\left(A_{T}\right)-g_{S, T}\left(B_{T}\right)+g_{S, T}\left(\theta_{T}\right) .
$$

Theorem 6. Let $S, T$ overlap and let $g_{S, T}$ be defined as in Def. 6. Then $g_{S, T}$ is a well defined, one-to-one mapping of $\bar{\sigma}_{T}$ onto $\vec{\Omega}_{S}$, satisfying the relations

$$
\begin{gathered}
g_{S, T} \overline{\left(\overline{A_{T}}, B_{T}\right)}=g_{S, T}\left(\theta_{T}\right)+h_{S} f_{S, T} h_{T}^{-1} \overline{\left(A_{T}, B_{T}\right)}, \\
g_{S, T}\left[\overline{\left(A_{1 T}, B_{1 T}\right)}+\overline{\left(A_{2 T}, B_{2 T}\right)}\right]=g_{S, T} \overline{\left(A_{1 T}, B_{1 T}\right)}+g_{S, T} \overline{\left(A_{2 T}, B_{2 T}\right)}-g_{S, T}\left(\theta_{T}\right) .
\end{gathered}
$$

Proof. Formula 2 follows immediately from (i) of Theorem 5, and shows that $g_{S, T}$ is well defined by Def. 6 and is one-to-one and onto, since it can be decomposed into a succession of mappings each of which is one-to-one and onto (see Theorem 2, Sec. 4). Formula 3 follows easily from (2) and the isomorphism properties of $h_{S} f_{S, T} h_{T}^{-1}$. 
Theorem 7. Suppose $S, T, U$ overlap. Then

(i) $g_{S, T}\left[g_{T, U}\left(\theta_{U}\right)\right]=g_{S, U}\left(\theta_{U}\right)$;

(ii) for all $A, B, g_{S, T}\left[g_{T, U\left(\overline{\left.A_{U}, B_{U}\right)}\right]}=g_{S, U} \overline{\left(A_{U}, B_{U}\right)}\right.$.

Proof. Choose $A, B, C$, such that $C^{U} \equiv B^{T} \equiv A^{s}$. By (ii) of Theorem 5 ,

By Eq. 3 (Theorem 6), one has

$$
g_{T, U}\left(\theta_{U}\right)=\overline{\left(B_{T}, \theta_{T}\right)}+h_{T} f_{T, U} h_{U}^{1} \overline{\left(\theta_{U}, \overline{C_{U}}\right)} .
$$

$$
g_{S, T}\left[g_{T, U}\left(\theta_{U}\right)\right]-g_{S, T} \overline{\left(B_{T}, \overline{\left.\theta_{T}\right)}\right.}+g_{S, T}\left[h_{T} f_{T, U} h_{U}^{-1} \overline{\left(\theta_{U}, \overline{C_{U}}\right)}\right] \quad g_{S, I}\left(\theta_{T}\right) .
$$

By (iii) of Theorem $\left.5, g_{S, T} \overline{(B} \bar{B}_{T}, \theta_{T}\right)=\overline{\left(A_{S}, \theta_{S}\right)}$, while by Eq. 2 of Theorem 6 ,

$$
\begin{aligned}
g_{S, T}\left[h_{T} f_{T, U} h_{U}^{-1}\left(\overline{\left.\theta_{U}, C_{U}\right)}\right]\right. & =g_{S, T}\left(\hat{\theta}_{T}\right)+h_{S} f_{S, T} h_{T}^{-1} h_{T} f_{T, U} h_{U}^{-1} \overline{\left(\theta_{U}\right.} \overline{\left.C_{U}\right)} \\
& =g_{S, T}\left(\theta_{T}\right)+h_{S} f_{S, U} h_{U}^{-1} \overline{\left(\theta_{U}, C_{U}\right)}
\end{aligned}
$$

(The last step utilizes Theorem 3 and the fact that $h_{T}^{-1} h_{T}$ drops out of the equation.) Combining these calculations yields

$$
\begin{aligned}
g_{S, T}\left[g_{T, U}\left(\theta_{U}\right)\right] & \left.=\overline{\left(A_{S}, \theta_{S}\right)}+h_{S} f_{S, U} h_{U}^{-1} \overline{\left(\theta_{U}\right.}, \overline{C_{U}}\right) \\
& =g_{S, U}\left(\theta_{U}\right)
\end{aligned}
$$

[again, using (ii) of Theorem 5 to get a convenient definition of $g_{S, U}\left(\theta_{U}\right)$ ]. This establishes statement (i) of the theorem.

Statcment (ii) can now be proved in a straightforward manner, expanding $g_{T, U} \overline{\left(A_{U}, B_{U}\right)}$ by (2) of Theorem 6 and applying (3) of Theorem 6 to the expansion, using statement (i) of the present theorem and Theorem 3 to calculate the components.

Q.E.D.

The preceding theorems show that, in fact, context changes can be described by means of transformations of groups of appearance differences, which preserve group structure: $g_{S, T}$ can be decomposed into an additive component, $g_{S, T}\left(\theta_{T}\right)$, and a transformation which preserves the additive binary operation, $h_{S} f_{S, T} h_{T}^{-1}$. The remarks concerning significance and data reduction made at the end of Sec. 4 apply here as well. The transformations $g_{S, T}$ will be called affine, since they exhibit properties analogous to those of affine geometry.

\section{STRUCTURE IN THE SET OF CONTEXTS}

In a sense, the pair of contexts $(S, U)$ is composed of the pairs $(S, T)$ and $(T, U)$; heuristically, one adds the latter two pairs componentwise and cancels $T$ from both sides, analogous to addition in the groups $\overline{\mathscr{Q}}_{S}$, etc. According to Theorems 3 and 7 , 
this "addition" of context pairs corresponds to composition of the corresponding functions $f$ or $g$; thus, the values of $f_{S, U}$ are predictable by composing $f_{S, T}$ and $f_{T, U}$. This suggests that much greater predictive power would result if more general ways of adding contexts or pairs of contexts were available, just as, for any one $f_{S, T}$ or $g_{S, T}$, predictions of some values result from knowledge of others via utilization of the group structure on $\overline{\mathscr{G}}_{T}$ or $\overline{\mathscr{C}}_{T}$.

The most obvious way to obtain group structure for pairs of contexts is to apply the construction of Sec. 2, to a commutative complete semi-group of one-to-one transformations of contexts. This is particularly applicable if the set of contexts under consideration corresponds in some natural way to the set of stimulus elements. For example, in the color domain, it is plausible to study a set of contexts in which all factors are held constant except for the color of a single homogeneous field, used for a preadapting stimulus or as part or all of a surrounding field. The sets $C$ and $\mathscr{S}$ then both correspond to the set of spectral energy distribution functions, and the same transformations may be appropriate for both stimulus elements and contexts.

In Assumption 5 below, it will be postulated that there is a semigroup $\mathscr{H}$ of transformations of $\mathscr{S}, s, t$, etc. To apply the construction of Sec. 2, the pairs $(S, T)$ and $\left(S^{s}, T^{s}\right)$ must be equivalent, so one will want to be able to conclude that $g_{S, T}=g_{S^{s}, T^{s}}$. Thus, in particular, if $A^{T} \equiv B^{S}$, then one must also have $A^{T^{s}} \equiv B^{S^{s}}$. Applying this to the case $S=T$, it follows that the relations $=s$ and $=s^{s}$ are identical, for all $S$ and $s$. By completeness of $\mathscr{H}$ with respect to $\mathscr{F}$, it follows that $=_{S}$ and $={ }_{T}$ are identical for all $S, T$. Thus, the situation discussed in Sec. 4 following Theorem 3 and its corollary obtains: there is one group $\overline{\mathscr{G}}$ of transformation differences, and one group $\overline{\mathscr{C}}$ of appearance differences; the functions $f_{S, T}$ are automorphisms of $\mathscr{G}$, while the $g_{S, T}$ are affine transformations of $\overline{\mathscr{C}}$ onto itself.

Assumption 5. (i) $\mathscr{H}$ is a commutative semigroup of one-to-one transformations of $\mathscr{S}$, which is complete with respect to $\mathscr{S}$.

(ii) For any $A, B, S, T$, and $s, A^{T} \equiv B^{S}$ if and only if $A^{T^{s}} \equiv B^{s^{s}}$.

Part (ii) means, in part, that the effect of transforming a context, on the appearance of the stimulus element, depends only on the original appearance of the stimulus element, not on the combination of stimulus and context that produces the original appearance. As mentioned above, this assumption has the particular consequence that stimuli which match in context $S$ also match in contcxt $T$, a consequence which is true for color matching for a wide range of conditions, as mentioned in Sec. 4.

Since the concepts of overlapping contexts, and the values of $f_{S, x}$ and $g_{S, T}$ depend ultimately on the cross-context matching relation $\equiv$, the following theorem is obvious.

TheOREM 8. For all $S_{1}, \ldots, S_{n}$ and $s$, contexts $S_{1}, \ldots, S_{n}$ overlap if and only if $S_{1}^{s}, \ldots, S_{n}^{s}$ overlap. If $S, T$ overlap, then $f_{S, T}=f_{S^{s}, T^{s}}$ and $g_{S, T}=g_{S^{s}, T^{s}}$. 
Theorem 8 has important corollaries. To state the corollaries it is convenient to use the group structure $\overline{\mathscr{S}}$ constructed as in Sec. 2, with elements $\overline{(S, T)}$, etc.

Corollary 1. If $S, T$ overlap and $U, V$ overlap, and $\overline{(S, T)}=\overline{(U, V)}$, then $f_{S, T}=f_{U, V}$ and $g_{S, T}=g_{U, V}$.

Proof. $\overline{(S, T)}=\overline{(U, V)}$ means that there exist $s, t$, such that $S^{s}=T^{t}$ and $U^{s}=V^{t}$. By completeness, there exist $u, v$ in $\mathscr{H}^{\prime}$ such that $S^{u}=U^{v}$.

Then

$$
\begin{aligned}
f_{S, T} f_{V, U} & =f_{S^{u t}, T^{u t}} f_{V^{n t}, U^{v t}} \\
& =f_{S^{u t},}, S^{u s} f_{U^{v s}, S^{u t}} \\
& =f_{S^{u t}, U^{v s}} f_{U^{v s}, S^{u t}} \\
& =f_{S^{u t}, S^{u t}} \\
& =\text { identity transformation. }
\end{aligned}
$$

The first step follows from Theorem 8 , the next two from commutativity of $\mathscr{H}$ and the properties of $s, t, u, v$; the fourth step follows from Theorem 3 , since $S^{u t}$ and $U^{v s}$ overlap by Theorem 8 ; and the last step follows from the corollary to Theorem 3 . Since $f_{S, T}$ is thus the inverse to $f_{V, U}$, by the corollary to Theorem 3 it follows that $f_{S, T}=f_{U, V}$. The argument for $g$ is analogous.

Q.E.D.

From Corollary 1, each element $\overline{(S, T)}$ in $\overline{\mathscr{P}}$ determines a single transformation $f_{\overline{(\bar{S}, \bar{T})}}$ or $g_{\overline{(S, T)}}$.

CoRollary 2. Suppose that $\overline{(S, T)}$ and $\overline{(U, V)}$ have the property that for any $S_{1}$, $S_{2}, S_{3}$ such that $\overline{\left(S_{1}, S_{2}\right)}=\overline{(S, T)}$ and $\overline{\left(S_{2}, S_{3}\right)}=\overline{(U, V)}, S_{1}, S_{2}, S_{3}$ overlap. (This property will be assumed below, whenever $S$ is sufficiently similar to $T$ and $U$ is sufficiently similar to $V$.) Then

$$
f_{\overline{(S, T)}+\overline{(U, V)}}=f_{\overline{(S, T)}} f_{(\overline{(U, V)}}
$$

and similarly for $g$.

Proof. Let $\overline{(S, T)}=\overline{\left(W^{s}, W^{t}\right)}$ and $\overline{(U, V)}=\overline{\left(W^{u}, W^{v}\right)}$.

Then $\overline{(S, T)}+\overline{(U, V)}=\overline{\left(W^{s u}, \overline{W^{t v}}\right)}$.

By Theorem 8 and by Corollary 1 above,

$$
\begin{aligned}
& f_{\overline{(S, T)}} f_{\overline{(U, V)}}=f_{\overline{\left(W^{s u}, W^{t u}\right)}} f_{\overline{\left(W^{t u}, W^{t}\right.}} \\
& =f \frac{f_{\left(W^{w u}, W^{t v}\right)}}{} .
\end{aligned}
$$


(The last step uses the fact that, by hypothesis, Theorem 3 is applicable, since $W^{s u}$, $W^{t u}$, and $W^{t v}$ overlap.) The argument for $g$ is exactly the same, using Theorem 7 instead of 3.

Q.E.D.

Corollary 2 shows that, if Assumption 5 is valid, predictions of appearance changcs for some pairs of contexts can be made on the basis of changes for other pairs, using the group structure $\overline{\mathscr{S}}$ obtained from transformations of contexts. Of course, these predictions are limited to the case where the pairs into which a given pair is decomposed involve sufficiently small context-differences so that the needed triple-overlap property is valid. The next section deals with the extension of the results of Sec. 4-6 to the case of dissimilar contexts.

\section{NONOVERLAPPING CON'TEXTS}

In this and the next section, the notation $\bar{A}, \bar{B}$, ctc. will be uscd for elements of $\bar{Q}$, and $\bar{S}, \bar{T}$, etc. will be used for elements of $\overline{\mathscr{S}}$. The zero elements of the groups $\overline{\mathscr{Q}}$ and $\overline{\mathscr{S}}$ will both be denoted $\theta$.

Under the conditions specified in Corollary 2 to Theorem 8 (Sec. 6), involving triple overlap, one has the equation

$$
g_{\bar{S}+\bar{T}}=g_{\bar{S}} g_{\bar{T}},
$$

and the question arises, whether this equation can be used to define the transformation $g_{\bar{S}+\bar{T}}$ in the case where $g_{\bar{S}}$ and $g_{\bar{T}}$ are defined but $g_{\bar{S}+\bar{T}}$ is not defined (pairs of contexts in $\bar{S}+\bar{T}$ do not overlap) or $\bar{S}, \bar{T}$ fail to satisfy the proper triple overlap condition. For such a definition to be possible, two requirements must be met: first, any $\bar{S}$ in $\overline{\mathscr{S}}$ must be decomposable into a sum, $\bar{S}=\bar{S}_{1}\left|\bar{S}_{2}\right| \cdots \mid \bar{S}_{m}$, where $\bar{S}_{1}, \ldots, \bar{S}_{m}$ all represent overlapping pairs of contexts; and second, it must be true that whenever $\bar{S}_{1}+\cdots+\bar{S}_{m}=\bar{T}_{1}+\cdots+\bar{T}_{n}$, where all the $\bar{S}_{i}$ and $\bar{T}_{j}$ represent overlapping context-pairs, then

$$
g_{S_{1}} g_{S_{2}} \cdots g_{\bar{S}_{m}}=\mathrm{g}_{\bar{T}_{1}} g_{\bar{T}_{2}} \cdots g_{\bar{T}_{n}} .
$$

At least, this last equation must hold for $\bar{S}_{i}, \bar{T}_{j}$ selected from some set sufficiently rich to define all the transformations $g_{S}$ in the above way.

The first requirement will be met by assuming that $\overline{\mathscr{P}}$ is a connected topological group (Assumption 6 below) such that for some neighborhood of $\theta$, any $S$ and $\bar{T}$ in the neighborhood satisfy the hypotheses of Corollary 2 to Theorem 8 , so that $g_{S}, g_{\bar{T}}$ and $g_{S+\bar{T}}$ are defined and $g_{\bar{S}} g_{\bar{T}}=g_{\bar{S}+\bar{T}}$. According to a theorem on topological groups (Pontrjagin, 1939, p. 76) every $\bar{S}$ in $\overline{\mathscr{S}}$ can be written in the form $\bar{S}_{1}+\cdots+\bar{S}_{m}$ for some $\bar{S}_{1}, \ldots, \bar{S}_{m}$ in such a neighborhood. (The theorem depends only on connectedness of the group.) 
The second requirement cited above can be proved to be satisfied, provided that $\overline{\mathscr{S}}$ satisfies certain other connectedness properties. Before stating the assumptions and theorems rigorously, I shall sketch a proof that the second requirement is satisfied, for one particular case; the method of proof carries over to the general theorems. For purposes of the proof, it is useful to think of the elements $\bar{S}$ as vectors, and of the addition as vector addition. Thus, a sum, $\bar{S}_{1}+\cdots+\bar{S}_{m}$ can be thought of as a polygonal line in space, with successive vertices at the origin (i.e., $\theta$ ), at $\bar{S}_{1}, \bar{S}_{1}+\bar{S}_{2}, \ldots$, and at $\bar{S}_{1}+\cdots+\bar{S}_{m}$. Two such polygonal lines are represented in Fig. 1 . I shall consider the case where $\bar{S}_{1}+\bar{S}_{2}+\bar{S}_{3}+\bar{S}_{4}=\bar{T}_{1}+\bar{T}_{2}+\bar{T}_{3}+\bar{T}_{1}$, depicted in the figure, with the polygonal lines meeting at the same endpoint. The figure also shows by dotted lines two "difference vectors" $\bar{C}_{2}=\bar{S}_{1}+\bar{S}_{2}-\bar{T}_{1}$, and $\bar{U}_{3}=\bar{S}_{1}+\bar{S}_{2}+\bar{S}_{3}-\bar{T}_{1}-\bar{T}_{2}$. It will be assumed first that the two polygonal lines are so close together, and the $\bar{S}_{i}$ and $\bar{T}_{j}$ involve such small steps, that the vectors $\bar{U}_{2}$ and $\bar{U}_{3}$ are also in the same neighborhood of $\theta$ (i.e., are sufficiently small) in which the hypotheses of Corollary 2 to Theorem 8 are satisfied. Now obviously, $\bar{U}_{3}+\bar{S}_{4}=$ $\bar{T}_{3}+\bar{T}_{4}$, and thus, by the "smallness" of these components,

$$
g_{\bar{U}_{3}} g_{S_{4}}=g_{\bar{U}_{3}+S_{4}}=g_{\bar{T}_{3}+\bar{T}_{4}}=g_{\bar{T}_{3}} g_{\bar{T}_{4}} .
$$

Similarly, $\bar{U}_{2}+\bar{S}_{3}=\bar{U}_{3}+\bar{T}_{2}$ and $\bar{U}_{2}+\bar{T}_{1}=\bar{S}_{1}+\bar{S}_{2}$, so that

$$
g_{\bar{U}_{2}} g_{S_{3}}=g_{\bar{U}_{3}} g_{\bar{T}_{2}} \quad \text { and } \quad g_{\sigma_{2}} g_{T_{1}}=g_{S_{1}} g_{S_{2}} \text {. }
$$

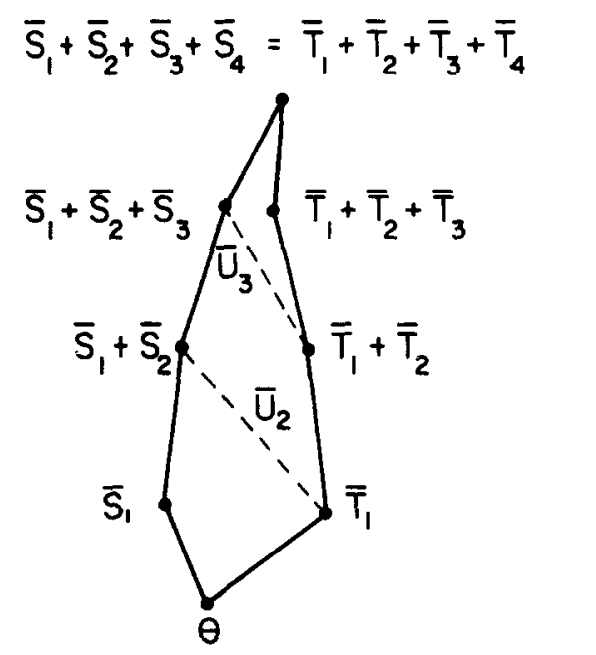

FIG. 1. Representation of two equal sums as polygonal lines. 
Multiplying together these equations, with all the $g_{S}$ terms on the left, yields,

$$
g_{S_{1}} g_{S_{2}} g_{\bar{U}_{2}} g_{S_{3}} g_{\bar{U}_{3}} g_{S_{4}}=g_{\bar{U}_{2}} g_{\bar{T}_{1}} g_{\bar{U}_{3}} g_{\bar{T}_{2}} g_{\bar{T}_{3}} g_{\bar{T}_{4}}
$$

Since the $g$ 's commute in pairs (again, because Corollary 2 of Theorem 8 holds for each pair) and are one-to-one (Theorem 6 ), the terms involving $\bar{U}_{2}$ and $\bar{U}_{3}$ can be cancelled from both sides, leaving the desired equality.

Clearly, the same process will work for any two sums of "small" terms whose polygonal lines lie close together. But sums of "large" terms can always be broken down into sums of small terms, and if two polygonal lines lie far apart, intermediate ones having the same endpoints may be inserted, according to the connectedness property that will be assumed. In fact, the above intuitive argument skirts one of the more profound ideas in mathematics. The essential property that between any two polygonal lines with the same endpoints one can insert many more polygonal lines with the same endpoints, as closely spaced as necessary, is called simple connectedness. The following basic theorem is proved in Pontrjagin (1939, p. $228 \mathrm{ff}$.):

Let $G_{1}$ and $G_{2}$ be topological groups, such that $G_{1}$ is locally connected and simply connected. Let $g$ be a local homomorphism of $G_{1}$ into $G_{2}$. Then there is a unique extension $g^{\prime}$ of $g$ into a homomorphism of the entire group $G_{1}$ into $G_{2}$.

Pontrjagin's theorem can be applied to the present case as follows: $G_{1}$ will be the group $\overline{\mathscr{P}}$, while $G_{2}$ will be the group $A(\overline{\mathscr{C}})$ of all affine transformations of $\overline{\mathscr{C}}$ onto itself. That is, $A(\overline{\mathscr{O}})$ consists of all one-to-one transformations of $\overline{\mathscr{O}}$ onto itself that satisfy Eq. 3 of Theorem 6 (Sec. 5), with the usual group operation for transformations. It is easy to show that the composition of two affine transformations is again affine, etc. The mapping $g$ of $\overline{\mathscr{S}}$ into $A(\overline{\mathscr{T}})$ simply sends $S$ into $g_{S}$. This will be a homomorphism in a neighborhood of $\theta$, i.e., where $g_{S+\bar{T}}=g_{S} g_{\bar{T}}$. The unique extension $g^{\prime}$, defined for all $\bar{S}$ in $\overline{\mathscr{S}}$, and coinciding with $g$ in a neighborhood of $\theta$, gives us the desired general definition of a transformation describing context changes, for all pairs of contexts. The precise assumptions needed can be stated as follows (see Pontrjagin, 1939, for all definitions).

Assumption 6. Topologies exist on $\overline{\mathscr{S}}$ and on $A(\overline{\mathscr{C}})$ such that

(i) $\overline{\mathscr{S}}$ and $A(\overline{\mathscr{C}})$ are topological groups, and the mapping $g$, sending $\bar{S}$ into $g_{S}$, is defined and continuous in a neighborhood of the identity $\theta$ of $\overline{\mathscr{S}}$;

(ii) $\overline{\mathscr{S}}$ is locally connected and simply connected;

(iii) for some neighborhood $\mathscr{V}$ of $\theta$ in $\overline{\mathscr{S}}$, any $\bar{S}, \bar{T}$, in $\mathscr{V}$ satisfy the hypotheses of Corollary 2 to Theorem 8, i.e., if $\overline{\left(S_{1}, S_{2}\right)}=\bar{S}$ and $\overline{\left(S_{2}, S_{3}\right)}=\bar{T}$, then $S_{1}, S_{2}, S_{3}$ overlap.

Part (iii) of Assumption 6 guarantees (by Corollary 2, Theorem 8) that the mapping $g$ is a local homomorphism of $\overline{\mathscr{S}}$ into $A(\overline{\mathscr{C}})$. Thus, Pontrjagin's theorem applies, yielding the following theorem: 
Theorem 9. There is one and only one way to assign, to each $\bar{S}$ in $\overline{\mathscr{P}}$, a transformation $g_{S}$ of $\overline{\mathscr{Q}}$, so that $g_{S}$ depends continuously on $\bar{S}, g_{S}$ coincides with the transformation defined by Defs. 5 and 6 (Sec. 5) in a neighborhood of $\theta$, and $g_{5}$ satisfies the equations

$$
\begin{aligned}
g_{\bar{S}}(\bar{A}+\bar{B}) & =g_{\bar{S}}(\bar{A})+g_{S}(\bar{B})-g_{\bar{S}}(\theta) \\
g_{S+\bar{T}}(\bar{A}) & =g_{S_{S}} g_{\bar{T}}(\bar{A}) .
\end{aligned}
$$

Theorem 9 was essentially proved in the preceding discussion. It should be remarked that the topological assumptions of Assumption 6 are not very restrictive. For example, if $\overline{\mathscr{S}}$ and $\overline{\mathscr{O}}$ are both finite-dimensional vector spaces, then there are unique natural topologies on $\overline{\mathscr{S}}$ and on $A(\overline{\mathscr{C}})$, the former is simply connected and locally connected, and continuity of the mapping $g$ is very natural; the only real content in the assumption is that for context-differences sufficiently close to zero, the triple overlap property is satisfied. The strength of the triple overlap property comes from the provision, in the definition of overlap, that for any two transformations $a, b$, a stimulus $A$ can be found such that not only $A^{T}$, but also $A^{a T}$ and $A^{b T}$ can be matched under the other two contexts, etc. That is, not only must $A^{T}$ be matchable, but $A$ must be "large" in comparison with the effects of $a$ and $b$, so that it remains in the portion of appearance space that can be matched, after transformation.

Note that the transformations $g_{S}$ of Theorem 9 do not necessarily coincide with those defined earlier for all $\bar{S}$, but only for context-differences that are sufficiently small. It is conceivable that some $\bar{S}, \bar{T}$, and $\bar{S}+\bar{T}$ might each represent overlapping pairs of contexts, but that triple overlap might fail. In such a case, there is no way to prove from the definitions that $g_{S+\bar{T}}=g_{S} g_{\bar{T}}$ and if, in fact, this is false, then at least one of the $g$ 's in Theorem 9 must differ from the one defined directly.

Equations 4 and 5 make full use of both group structures, on $\overline{\mathscr{G}}$ and on $\overline{\mathscr{F}}$, to predict some appearance changes from others. The exact degree of data reduction and prediction depends on what further assumptions can be made regarding the generation of some elements of $\overline{\mathscr{C}}$ and $\overline{\mathscr{T}}$ as sums of others. In the next section, one such possibility is explored, namely, that $\overline{\mathscr{O}}$ and $\overline{\mathscr{S}}$ are three-dimensional vector spaces. This may be applicable in the case of color vision; in any case, it is the example from which the theory in the preceding sections was obtained by generalization.

\section{THE FUNCTIONAL EQUATIONS FOR ADAPTATION AND CONTRAST EFFECTS IN COLOR SPACE}

In this section, stimulus clcmcnts, $A, B$, etc, and contexts, $S, T$, etc. are identified with particular spectral energy distribution functions. A stimulus element is assumed to consist of a small homogeneous patch of light, viewed foveally with one eye, with all viewing conditions fixed except for the wavelength composition of a homogeneous 
preadapting or surround field; variations in the latter constitute variations in context. The relations $=s$ are assumed to be identical equivalence relations, namely, the relation of metameric pairs. The experimental arrangements mentioned in Sec. 3, in connection with Assumption 1, will be assumed to hold, so that $\equiv$ is an equivalence relation. The transformations in $\mathscr{G}$ and $\mathscr{H}$ consist of addition of fixed distributions, either to stimulus elements, in the case of $\mathscr{G}$, or to contexts, in the case of $\mathscr{H}$. The transformation semigroup $\mathscr{G}$ is, of course, assumed to be context-invariant with respect to $\mathscr{P}$.

If $\theta$ denotes the spectral energy distribution with zero energy density everywhere, then the transformation that involves adding $A$ can be identified with the element $\theta^{A}=\theta+A=A$, and similarly for $S$. Thus, the group $\overline{O l}$ consists of classes $\overline{(A, B)}$, with $\overline{\left(A_{1}, B_{1}\right)}=\overline{\left(A_{2}, B_{2}\right)}$ if and only if $A_{1} \mid B_{2}$ is metameric to $A_{2} \mid B_{1}$, and addition of such classes corresponds to componentwise additive color mixture. This addition is well defined because of part (ii) of Assumption 2, which, as pointed out in Sec. 3, is a special case of Grassman's Third Law.

The special feature of color stimuli is that the group $\overline{\mathscr{C}}$ can be made into a vector space in a natural way, by introducing scalar multiplication. For any nonnegative real number $p$, one may define $p A$ to be the stimulus whose spectral energy distribution is equal to that of $A$, multiplied by a factor $p$; i.e., the energy density at each wavelength is multiplied by $p$. Thus, $0 A=\theta$ and $1 A=A$. Moreover,

$$
\begin{aligned}
p(A+B) & =p A+p B, \\
(p+q) A & =p A+q A, \\
(p q) A & =p(q A) .
\end{aligned}
$$

These properties involve only physics. Finally, there is one psychological property of scalar multiplication, which is closely related to Grassman's Laws: for any $p, A, B, S$,

$$
A^{S}=B^{S} \text { if and only if }(p A)^{S} \equiv(p B)^{S} .
$$

This is valid for a wide range of positive values of $p$ (see Judd, 1951). Similar considerations hold, in this case, for the contexts $S$.

One can define scalar multiplication in $\bar{Q}$ as follows: $p \overline{(A, B)}=\overline{(p A, p B)}$ if $p$ is nonnegative; $p \overline{(A, B)}=(-p) \overline{(B, A)}$ if $p$ is negative. The properties stated above are sufficient to imply that scalar multiplication in $\bar{Q}$ is well defined and that $\bar{O}$ is a vector space. Similarly, $\overline{\mathscr{S}}$ can be made into a vector space.

In the color literature, the vectorial representation of metamer-classes of stimuli has long been understood. It has been achieved in terms of coordinate systems derived from three-primary matching. Actually, the vectorial representation depends essentially on Grassman's Third Law and not at all upon primaries; only the dimensionality of the vector space is determined by three-primary matching. 
The facts of trichromacy of color vision can be easily stated in these terms: for a normal human observer, the vector space $\overline{\mathscr{C}}$ is three-dimensional. (Similarly, $\overline{\mathscr{S}}$ is threedimensional.)

The tri-dimensionality of these spaces is a consequence of the following empirical facts: (i) if any 4 spectral energy distribution functions are chosen, they may be divided into 2 non-empty sets, and suitable nonnegative scalar multiples of them chosen, not all $=\theta$, so that the sum of the scalar multiples of the distribution functions in one set, and the same sum for the other set, are metamers; (ii) the analogous statement for 3 spectral energy distribution functions is false, for someone with normal color vision. (An observer with a two-dimensional space is called a dichromat; one with a onedimensional space is a monochromat (totally color-blind)).

If three suitable primaries $\bar{A}_{1}, \bar{A}_{2}, \bar{A}_{3}$ are chosen in $\overline{\mathscr{A}}$, then every element $\bar{A}$ can be expressed in the form

$$
\bar{A}=p_{1} \bar{A}_{1}+p_{2} \bar{A}_{2}+p_{3} \bar{A}_{3},
$$

where the $p$ 's are any real numbers. The condition for suitability is merely that none of the $\bar{A}_{i}$ can be so expressed in terms of the other two. Similarly, $\bar{S}_{1}, \bar{S}_{2}, \bar{S}_{3}$ can be chosen in $\overline{\mathscr{P}}$ such that every $\bar{S}$ can be written as such a linear combination of the $\bar{S}_{i}$. Assuming that the functions $g_{S}$ are continuous, Eq. 4 implies that

$$
g_{S}(p \bar{A})=p g_{S}(\bar{A})+(1-p) g_{S}(\theta) .
$$

(See, for example, Hille and Phillips, 1957, p. 144.) Thus, one has

$$
g_{S}(\bar{A})=p_{1} g_{S}\left(\bar{A}_{1}\right)+p_{2} g_{S}\left(\bar{A}_{2}\right)+p_{2} g_{S}\left(\bar{A}_{3}\right)+\left(1-p_{1}-p_{2}-p_{3}\right) g_{S}(\theta) ;
$$

so that from knowledge of 12 numbers, namely, the coordinates (in terms of $\bar{A}_{1}$, $\left.\bar{A}_{2}, \bar{A}_{3}\right)$ of $g_{S}\left(\bar{A}_{1}\right), g_{S}\left(\bar{A}_{2}\right), g_{S}\left(\bar{A}_{3}\right)$, and $g_{S}(\theta)$, one can predict the values of $g_{S}(\bar{A})$ for all $\bar{A}$. Similarly, it turns out that one can predict all the functions $g_{S}$ from knowledge of $g_{\bar{S}_{1}}, g_{S_{2}}$, and $g_{\bar{S}_{3}}$, and relations of form

$$
\bar{S}=p_{1} \bar{S}_{1}+p_{2} \bar{S}_{2}+p_{3} \bar{S}_{3} .
$$

The manner of making such predictions will be explained below; it requires taking a "logarithm" of the transformation $g_{S}$. At any rate, this means that all predictions of changes in appearance with changes in context can be made, on the basis of the above theory, from knowledge of at most 36 parameters; and it will be seen that this number can be reduced still further.

The remainder of this section is devoted to the problem of specifying all continuous solutions to Eqs. 4 and 5, which are assumed valid for all $\bar{A}, \bar{B}, \bar{S}, \bar{T}$ in the finitcdimensional vector spaces $\overline{\mathscr{q}}$ and $\overline{\mathscr{S}}$. The method of solution does not depend on the number of dimensions, but details will be specified only for the case when both spaces are three-dimensional. 
The solution has two stages. In the first stage, Eqs. 4 and 5 are rewritten in terms of the transformations $f_{S}$ :

$$
f_{S}(\bar{A})=g_{S}(\bar{A})-g_{S}(\theta)
$$

These transformations $f_{S}$ are practically the same as those denoted " $f$ " in Secs. 4-6 (compare Eq. 2, Sec. 5) except that they operate in $\bar{O}$ rather than in $\overline{\mathscr{G}}$, including the effects of $h_{S}, h_{T}^{-1}$ from Eq. 2 . In the second stage of the solution, the possible values of $g_{S}(\theta)$ are determined.

In terms of the $f^{\prime}$ s defined above, Eqs. 4 and 5 become

$$
\begin{aligned}
f_{\bar{S}}(\bar{A}+\bar{B}) & =f_{\bar{S}}(\bar{A})+f_{\bar{S}}(\bar{B}) \\
f_{S+\bar{T}}(\bar{A}) & =f_{S} f_{\bar{T}}(\bar{A}) .
\end{aligned}
$$

Equation $4^{\prime}$ simply asserts that the $f_{S}$ are linear transformations in the vector space $\bar{O}$. [As remarked above in connection with the $g$ 's, continuity guarantees that $f_{S}(p \bar{A})=p f_{\bar{S}}(\bar{A})$, the other requirement for linearity besides Eq. 4'. See Jacobson, 1953 , for definitions of terms in connection with vector spaces and linear transformations.]

Let $f$ be any linear transformation of a finite dimensional vector space into itself. Denote by $f^{n}$ the composition of $f$ with itself $n$ times, i.e., $f^{2}(\bar{A})=f(f(\bar{A}))$, etc. Let $\exp (f)$ be defined by

$$
\begin{aligned}
\exp (f)(\bar{A})=\bar{A}+(1 / 1 !) f(\bar{A}) & +(1 / 2 !) f^{2}(\bar{A})+\cdots \\
& +(1 / n !) f^{n}(\bar{A})+\cdots
\end{aligned}
$$

the sum being convergent in the vector space of linear transformations of the original space. It is readily shown that $\exp (f)$ is a linear transformation, and that if $f_{1}$ and $f_{2}$ commute, then $\exp \left(f_{1}+f_{2}\right)=\exp \left(f_{1}\right) \exp \left(f_{2}\right)$.

Let $\bar{S}_{1}, \bar{S}_{2}, \bar{S}_{3}$ be linearly independent vectors in $\overline{\mathscr{P}}$, and let $f_{1}, f_{2}$, and $f_{3}$ be arbitrary, pairwise-commutative linear transformations of $\bar{\emptyset}$ into itself. If $\bar{S}=p_{1} \bar{S}_{1}+p_{2} \bar{S}_{2}+p_{3} \bar{S}_{3}$, define a linear transformation $f_{S}^{*}$ by

$$
f_{\bar{S}}^{*}(\bar{A})=p_{1} f_{1}(\bar{A})+p_{2} f_{2}(\bar{A})+p_{3} f_{3}(\bar{A}) .
$$

Now define $f_{S}=\exp \left(f_{S}^{*}\right)$. Since $f_{S+\bar{T}}^{*}=f_{S}^{*}+f_{\bar{T}}^{*}$, it follows that $f_{S}$ satisfies Eq. $5^{\prime}$, hence, gives a solution for Eqs. $4^{\prime}$ and $5^{\prime}$.

According to a theorem of Hille and Phillips (1957, p. 290) all continuous solutions of Eqs. 4' and $5^{\prime}$ are obtained in this way. This completes stage 1 of the solution of (4) and (5). From knowledge of $f_{S_{1}}, f_{\bar{S}_{2}}, f_{S_{3}}$ (which can be more or less arbitrary-they must be one-to-one), it is possible to infer the values of the "logarithms" $f_{1}, f_{2}$, and $f_{3}$, and thus, by taking linear combinations and exponentiating, one obtains any $f_{\bar{S}}$. 
Now the possible values of $g_{S}(\theta)$ will be investigated. For each $\bar{S}, g_{S}(\theta)$ is a vector in $\overline{\mathscr{T}}$, so that a function $g: g(\bar{S})=g_{S}(\theta)$ is determined, mapping $\overline{\mathscr{S}}$ into $\overline{\mathscr{T}}$. Define linear transformations $h_{S}$ by $h_{S}(\bar{A})=f_{S}(\bar{A})-\bar{A}$. Then $g$ and $h_{S}$ satisfy the following functional equations:

$$
\begin{gathered}
h_{S+\bar{T}}=h_{\bar{S}} h_{\bar{T}}+h_{S}+h_{\bar{T}} \\
g(\bar{S}+\bar{T})-g(\bar{S})-g(\bar{T})=h_{S}(g(\bar{T}))=h_{\bar{T}}(g(\bar{S})) .
\end{gathered}
$$

These equations can easily be derived by replacing the left and right hand sides by their definitions in terms of $f_{\bar{S}}, f_{\bar{T}}$, and $f_{\bar{S}+\bar{T}}$, and using $\left(4^{\prime}\right)$ and $\left(5^{\prime}\right)$ to simplify the resulting expressions. From $\left(5^{\prime \prime}\right)$ and (6) and the linearity of the $h_{\bar{S}}$, two facts are apparent: first, for any fixed vector $\bar{A}$ in $\bar{q}$, the function $g(\bar{S})=h_{S}(\bar{A})$ yields a possible solution to Eq. 6 , and second, the difference between any two solutions to Eq. 6 is again a solution. These facts lead to the reduction of the problem of finding solutions of Eq. 6 to finding solutions such that the values of $g(\bar{S})$ are contained in a special subspace, $\mathscr{N}_{\infty}$, of $\overline{\mathscr{d}}$.

For each $\bar{S}$ in $\overline{\mathscr{S}}$, let $\mathscr{N}_{S}$ be the set of all vectors $\bar{A}$ in $\overline{\mathscr{R}}$ such that $h_{S}^{m}(\bar{A})=\theta$ for some positive integer $m$. $\left(\mathscr{N}_{S}\right.$ is called the null-component of $\left.h_{S}.\right)$ Let $\mathscr{N}_{\infty}$ be the intersection of all the $\mathscr{N}_{\bar{S}}$. Each $\mathscr{N}_{S}$ is a vector subspace of $\overline{\mathscr{C}}$, so $\mathscr{N}_{\infty}$ is also.

TheOREM 10. If $g$ is a solulion of Eq. 6 , then there exists a vector $\bar{A}_{\infty}$ in $\overline{\mathscr{O}}$ and a function $g_{\infty}$ from $\overline{\mathscr{T}}$ to $\mathscr{N}_{\infty}$, such that $g_{\infty}$ is also a solution to (6) and such that

$$
g(\bar{S})=g_{\propto}(\bar{S})+h_{S}\left(\bar{A}_{\propto}\right) .
$$

Theorem 10 shows that every solution of (6) can be obtained as a sum of a solution of form $h_{S}\left(\bar{A}_{\infty}\right)$ and a solution with values in $\mathscr{N}_{\infty}$. In particular, if $\mathscr{N}_{\infty}$ reduces to $\theta$, then the only solutions to (6) have form $h_{S}\left(\bar{A}_{\infty}\right)$.

Proof. The null-component $\mathscr{N}_{S}$ of $h_{S}$ was defined above. The rank-component $\mathscr{R}_{S}$ is defined to be the set of $\bar{A}$ such that for every positive integer $m$, there exists some $\bar{B}$ with $h_{S}^{m}(\bar{B})=\bar{A}$. According to Fitting's Lemma (Jacobson, 1951, p. 155; and 1953, p. 47), the linear transformation $h_{S}$ maps $\mathscr{N}_{S}$ and $\mathscr{R}_{S}$ into themselves, and every vector $\bar{A}$ can be written in a unique way as a sum of an element in $\mathscr{N}_{S}$ and an element in $\mathscr{R}_{S}$. Moreover, it is easy to prove that, when two transformations commute, either maps the null- and the rank-components of the other into themselves.

For any fixed $\bar{S}_{1}$, one can split all the vectors $g(S)$ into the sum of an element $g_{11}(\bar{S})$ in $\mathscr{N}_{S_{1}}$ and an element $g_{12}(\bar{S})$ in $\mathscr{R}_{S_{1}}$. By definition of the rank-component, there exists some $\bar{A}_{1}$ in $\mathscr{R}_{\bar{S}_{1}}$ such that $g_{12}\left(\bar{S}_{1}\right)=h_{S_{1}}\left(\bar{A}_{1}\right)$. Since by commutativity, any $h_{S}$ maps $\mathscr{R}_{S_{1}}$ into itself, it follows that $h_{S}\left(\bar{A}_{1}\right)$ is in $\mathscr{R}_{S_{1}}$ for every $S$. Now it will be shown that $g(\bar{S})-h_{S}\left(\bar{A}_{1}\right)$ is in $\mathscr{N}_{\bar{S}_{1}}$ for every $\bar{S}$. This will show that for every $\bar{S}$,

$$
g_{12}(\bar{S})=h_{S}\left(\bar{A}_{1}\right)
$$


because the decomposition of $g(\bar{S})$ into null-component and rank-component elements is unique. To prove that $g(\bar{S})-h_{S}\left(\bar{A}_{1}\right)$ is in the null-component of $h_{S_{1}}$, note first that there exists some integer $n$ (in the present case, $n$ can be taken $=3$ ) such that for every $\bar{A}$ in $\mathscr{N}_{S_{1}}, h_{S_{1}}^{n}(\bar{A})=\theta$. This is true from dimensionality arguments. In particular

$$
\begin{aligned}
h_{S_{1}}^{n}\left[g\left(\bar{S}_{1}\right)\right] & =h_{S_{1}}^{n}\left[g_{11}\left(\bar{S}_{1}\right)+g_{12}\left(\bar{S}_{1}\right)\right] \\
& \left.=h_{S_{1}}^{n}\left[g_{12}\left(\bar{S}_{1}\right)\right] \quad \text { (since } g_{11}\left(\bar{S}_{1}\right) \text { is in } \mathscr{N}_{S_{1}}\right) \\
& =h_{S_{1}}^{n+1}\left(\bar{A}_{1}\right) \quad \text { (by substitution) }
\end{aligned}
$$

Using this result, and Eq. 6, plus the commutativity of $h_{S}$, it can be shown that

$$
h_{S_{1}}^{n+1}\left[g(\bar{S})-h_{S}\left(\bar{A}_{1}\right)\right]=\theta .
$$

In fact,

$$
\begin{aligned}
h_{S_{1}}^{n+1}\left[g(\bar{S})-h_{S}\left(\overline{A_{1}}\right)\right] & =h_{S_{1}}^{n+1}[g(\bar{S})]-h_{S_{1}}^{n+1} h_{S}\left(\bar{A}_{1}\right) \\
& =h_{S_{1}}^{n} h_{S_{1}}[g(\bar{S})]-h_{S} h_{S_{1}}^{n+1}\left(\bar{A}_{1}\right) \\
& =h_{S_{1}}^{n} h_{S}\left[g\left(\bar{S}_{1}\right)\right]-h_{S} h_{S_{1}}^{n}\left[g\left(\bar{S}_{1}\right)\right] \\
& =\theta .
\end{aligned}
$$

It follows that $g(\bar{S})-h_{S}\left(\bar{A}_{1}\right)$ is in $\mathscr{N}_{S_{1}}$, and thus that for every $\bar{S}, g_{12}(\bar{S})=h_{S}\left(\bar{A}_{1}\right)$, or $g(\bar{S})=g_{11}(\bar{S})+h_{S}\left(\bar{A}_{1}\right)$. Since $g_{11}$ is the difference of two solutions to Eq. 6, it is again a solution, and since, for any $\bar{S}, h_{\bar{S}}$ can be regarded as a linear transformation of $\mathcal{N}_{S_{1}}$ into itself, with $g_{11}$ a solution of (6) taking values in $\mathcal{N}_{S_{1}}$, the same process can be repeated, within $\mathscr{N}_{S_{1}}$, that was just carried out within $\widetilde{a r}$, resulting in a decomposition of $g_{11}$ as $g_{11}(\bar{S})=g_{21}(\bar{S})+h_{S}\left(\bar{A}_{2}\right)$ where $g_{21}$ is another solution of (6), taking values in the intersection of $\mathscr{N}_{S_{1}}$ and $\mathscr{N}_{S_{2}}$, where $\bar{S}_{2}$ is another vector in $\overline{\mathscr{P}}$. By the finite dimensionality, this process must eventually yield a solution $g_{r 1}$ of (6) whose values are in $\mathscr{N}_{\infty}$; in this case, if $\bar{S}_{i}$ is chosen at the $i$ th step so as to decrease the dimensionality of the intersection of null-components, the process must stop after 4 steps, at most. If one denotes the final $g_{r 1}$ by $g_{\infty}$, and defines $\bar{A}_{\infty}$ to be the sum of the $\bar{A}_{i}$, one obtains Eq. 7, as required by the theorem.

Q.E.D.

To complete the determination of all possible solutions to Eq. 6, it is necessary to determine the solutions that take values in $\mathscr{N}_{\infty}$. If the linear transformations $h_{S}$ are regarded as transformations of $\mathscr{N}_{\infty}$ into itself, they form a commutative set of nilpotent transformations, and according to standard theorems (see Jacobson, 1953, p. 133-134) one can choose a basis for $\mathscr{N}_{\infty}$ such that the matrices of the transformations $h_{S}$ with 
respect to that basis have zeros on and above the main diagonal. In the present case, if the dimension of $\mathscr{N}_{\infty}$ is $=3$, one can choose linearly independent vectors $\bar{B}_{1}, \bar{B}_{2}$, $\bar{B}_{3}$ in $\mathscr{N}_{\infty}$ such that for all $\bar{S}$

$$
\begin{aligned}
& h_{S}\left(\bar{B}_{1}\right)=\theta, \\
& h_{S}\left(\bar{B}_{2}\right)=h_{21}(\bar{S}) \bar{B}_{1}, \\
& h_{S}\left(\bar{B}_{3}\right)=h_{31}(\bar{S}) \bar{B}_{1}+h_{32}(\bar{S}) \bar{B}_{2},
\end{aligned}
$$

where the functions $h_{21}, h_{31}, h_{32}$ are real-valued functions defined on $\overline{\mathscr{P}}$. If the dimension of $\mathscr{N}_{\infty}$ is 2 or 1 , only the first two or one of these equations is relevant, while if the dimension is 0 , there are no nontrivial solutions to (6) with values in $\mathscr{N}_{\infty}$. Furthermore, the functions $h_{i j}$ must satisfy, by commutativity, the relation

$$
h_{32}(\bar{S}) h_{21}(\bar{T})=h_{32}(\vec{T}) h_{21}(\bar{S})
$$

for all $\bar{S}, \bar{T}$. Equation 9 implies that the functions $h_{32}$ and $h_{21}$ are proportional (unless one of them is identically zero). (In the general solution, for dimensions greater than 3 , commutativity similarly imposes a series of relations on the $h_{i j}$, which permits specification of all of them except for arbitrary constants, in terms of one (which is nonidentically zero) from each diagonal parallel to the main diagonal.) In terms of the $\bar{B}_{i}$, any solution $g$ to Eq. 6 with values in $\mathscr{N}_{\infty}$ can be written in the form

$$
g(\bar{S})=g_{1}(\bar{S}) \bar{B}_{1}+g_{2}(\bar{S}) \bar{B}_{2}+g_{3}(\bar{S}) \bar{B}_{3},
$$

where the $g_{i}$ are real-valued functions defined on $\overline{\mathscr{S}}$. (In cases of dimension less than 3 , the latter terms of Eq. 10 are omitted.) Equation (6) now gives three equal expressions in $\mathscr{N}_{\infty}$, which can each be written in terms of the $\bar{B}_{i}$, and the coefficients of the $\bar{B}_{i}$ must then be equal. From equating coefficients of $\bar{B}_{3}$ one obtains

$$
g_{3}(\bar{S}+\bar{T})-g_{3}(\bar{S})-g_{3}(\bar{T})=0 .
$$

From equating coefficients of $\bar{B}_{2}$ one has

$$
\begin{aligned}
g_{2}(\bar{S}+\bar{T})-g_{2}(\bar{S})-g_{2}(\bar{T}) & =h_{32}(\bar{S}) g_{3}(\bar{T}) \\
& =h_{32}(\bar{T}) g_{3}(\bar{S})
\end{aligned}
$$

while from the coefficients of $\bar{B}_{1}$ one has

$$
\begin{aligned}
g_{1}(\bar{S}+\bar{T})-g_{1}(\bar{S})-g_{1}(\bar{T}) & =h_{31}(\bar{S}) g_{3}(\bar{T})+h_{21}(\bar{S}) g_{2}(\bar{T}) \\
& =h_{31}(\bar{T}) g_{3}(\bar{S})+h_{21}(\bar{T}) g_{2}(\bar{S})
\end{aligned}
$$

Note that for dimension of $\mathscr{N}_{\infty}=2$, Eq. 11 drops out while (12) reduces to (11) since the right-hand side is 0 , and (13) reduces to (12). For dimension of $\mathscr{N}_{\infty}=1$, only (13) is relevant and it reduces to (11). 
Under the assumption of continuity, these three functional equations may easily be solved. For simplicity, it will be assumed that the dimension is 3 and that none of the $h_{i j}$ is identically zero. The solutions for dimensions 2 or 1 follow immediately, while the cases where some of the $h_{i j}$ are identically zero can be treated separately by the same methods.

First, Eq. 11 implies that $g_{3}$ is a linear function from $\overline{\mathscr{P}}$ to the real numbers. Equation 12 implies that $h_{32}$ (and hence, $h_{21}$, from the previous discussion) is proportional to $g_{3}$. Thus, $h_{32}$ and $h_{21}$ must be linear in order for (6) to have any solutions in this case. Let $h_{32}=q_{1} g_{3}$, where $q_{1}$ is the proportionality constant. Then from (12) one has

$$
g_{2}(\bar{S}+\bar{T})=g_{2}(\bar{S})+g_{2}(\bar{T})+q_{1} g_{3}(\bar{S}) g_{3}(\bar{T}) .
$$

By inspection, this equation has the general solution

$$
g_{2}(\bar{S})=(1 / 2) q_{1}\left[g_{3}(\bar{S})\right]^{2}+\ell_{1}(\bar{S}),
$$

where $\ell_{1}$ is an arbitrary linear real-valued function on $\overline{\mathscr{S}}$. It is easy to prove that this solution is unique: if one defines a function $\ell_{1}$ by Eq. 14 , then $\left(12^{\prime}\right)$ implies that $\ell_{1}$ satisfies Eq. 11, hence, is linear.

Let $q_{2}$ be a proportionality constant such that $h_{21}=q_{2} g_{3}$. Then substituting for $h_{21}$ and for $g_{2}$ (using Eq. 14) in the right-hand parts of Eq. 13 yields the equation

$$
\begin{aligned}
& h_{31}(\bar{S}) g_{3}(\bar{T})+(1 / 2) q_{1} q_{2} g_{3}(\bar{S})\left[g_{3}(\bar{T})\right]^{2}+q_{2} g_{3}(\bar{S}) \ell_{1}(\bar{T}) \\
& \quad=h_{31}(\bar{T}) g_{3}(\bar{S})+(1 / 2) q_{1} q_{2} g_{3}(\bar{T})\left[g_{3}(\bar{S})\right]^{2}+q_{2} g_{3}(\bar{T}) \ell_{1}(\bar{S}) .
\end{aligned}
$$

The variables $\bar{S}$ and $\bar{T}$ can be separated in (15) by combining all terms containing $g_{3}(\bar{S})$ to the first power on one side, all terms containing $g_{3}(\bar{T})$ to the first power on the other, and dividing through both sides of the equation by $g_{3}(\bar{S}) g_{3}(\bar{T})$. This leads to the conclusion that $h_{31}(\bar{S})-(1 / 2) q_{1} q_{2}\left[g_{3}(\bar{S})\right]^{2}-q_{2} \ell_{1}(\bar{S})$ is proportional to $g_{3}(\bar{S})$. Call the proportionality constant $q_{3}$; then one can solve for $h_{31}$, obtaining

$$
h_{31}(\bar{S})=(1 / 2) q_{1} q_{2}\left[g_{3}(\bar{S})\right]^{2}+q_{3} g_{3}(\bar{S})+q_{2} \ell_{1}(\bar{S}) .
$$

When this solution is substituted into the right side of Eq. 13, along with the previous substitutions that lead to (15), one obtains the functional equation

$$
\begin{aligned}
g_{1}(\bar{S}+\bar{T})= & g_{1}(\bar{S})+g_{1}(\bar{T})+q_{2}\left[\ell_{1}(\bar{S}) g_{3}(\bar{T})+\ell_{1}(\bar{T}) g_{3}(\bar{S})\right]+q_{3} g_{3}(\bar{S}) g_{3}(\bar{T}) \\
& +(1 / 2) q_{1} q_{2}\left\{\left[g_{3}(\bar{S})\right]^{2} g_{3}(\bar{T})+\left[g_{\mathrm{s}}(\bar{T})\right]^{2} g_{3}(\bar{S})\right\}
\end{aligned}
$$

By inspection, $\left(13^{\prime}\right)$ has the general solution

$$
g_{1}(\bar{S})=(1 / 6) q_{1} q_{2}\left[g_{3}(\bar{S})\right]^{3}+(1 / 2) q_{3}\left[g_{3}(\bar{S})\right]^{2}+q_{2} g_{3}(\bar{S}) \ell_{1}(\bar{S})+\ell_{2}(\bar{S})
$$


where $\ell_{2}$ is a second arbitrary linear real-valued function on $\overline{\mathscr{T}}$. Uniqueness is proved in the same way: if (17) is used to define a function $\ell_{2}$, then $\left(13^{\prime}\right)$ can be used to prove that it is linear.

Equations 14 and 17 , together with (10), give a complete specification of possiblc solutions $g$ to Eq. 6, taking values in a three-dimensional $\mathscr{N}_{x}$, in terms of 3 arbitrary linear functions, $t_{1}, \ell_{2}$, and $g_{3}$, and three constants, $q_{1}, q_{2}$, and $q_{3}$. For a twodimensional $\mathscr{N}_{\infty}, g_{2}$ is linear, and $g_{1}$ is given in terms of $g_{2}$ by (14), with the numbers 2 and 1 substituted for 3 and 2 . For a one-dimensional $\mathscr{N}_{\infty}, g_{1}$ must be linear.

I shall now summarize the above results by presenting the possible functions $g_{S}$ that satisfy the functional equations (4) and (5). There are 4 cases to be distinguished, according to whether the dimension of $\mathscr{N}_{\alpha}$ is $0,1,2$, or 3 .

CASE 1. Dimension of $\mathscr{N}_{\infty}=0$.

Let $\bar{S}_{1}, \bar{S}_{2}, \bar{S}_{3}$ be any three linearly independent vectors in $\overline{\mathscr{S}}$. Choose three pairwise commutative linear transformations $f_{1}, f_{2}, f_{3}$ of $\overline{\mathscr{O}}$, whose null-components in $\bar{O}$ contain only $\theta$ in their common intersection. If $\bar{S}=p_{1} \bar{S}_{1}+p_{2} \bar{S}_{2}+p_{3} \bar{S}_{3}$, define

$$
\begin{aligned}
f_{S}^{*} & =p_{1} f_{1}+p_{2} f_{2}+p_{3} f_{3} \\
f_{S} & =\exp \left(f_{S}^{*}\right) \\
h_{S}(\bar{A}) & =f_{S}(\bar{A})-A .
\end{aligned}
$$

Let $\bar{A}_{\infty}$ be an arbitrary vector in $\overline{\mathscr{r}}$ and define $g(\bar{S})=h_{S}\left(\bar{A}_{\infty}\right)$. Now define

$$
g_{S}(\bar{A})=f_{S}(\bar{A})+g(\bar{S}) .
$$

The transformations $g_{S}$ defined in this way satisfy (4) and (5), with the dimension of $\mathscr{N}_{\infty}=0$, and every such set of solutions is obtained by the above construction, by varying the choice of $f_{1}, f_{2}, f_{3}$, and $\bar{A}_{\infty}$. These statements all follow from the previous results, provided one can show that $\mathscr{N}_{\infty}$ has dimension 0 if and only if the null components of $f_{1}, f_{2}$ and $f_{3}$ intersect in $\theta$. This latter fact follows from the fact that the null-components of $h_{S}$ and $f_{S}^{*}$ coincide, which can be proved fairly readily from considerations of the formulae for logarithms and exponentials of linear transformations. Thus, $\mathscr{N}_{\infty}$ has dimension 0 if and only if the null-components of the $f_{S}^{*}$ have only $\theta$ in common. But if there is a vector $\neq \theta$ in the null-components of $f_{1}, f_{2}$, and $f_{3}$, then by commutativity, it is in the null-component of every $f_{S}^{*}$ and conversely, any vector in the null-component of every $f_{S}^{*}$ is, in particular, in the null-components of the $f_{i}$, since $f_{i}=f_{S_{i}}^{*}$.

Note that all the $g_{S}$ arc defincd by choice of 30 parameters: the three $3 \times 3$ matrices of the $f_{i}$, and the three coordinates of $A_{\infty}$. This represents a reduction over the figure of 36 mentioned at the beginning of this section; the reduction is effected by Theorem 10, which expresses all the $g_{S}(\theta)$ in terms of $\bar{A}_{\infty}$. 
Note that in the present case, $g_{S}\left(-\bar{A}_{\infty}\right)=-\bar{A}_{\infty}$. Thus, there is a vector, $-\bar{A}_{\infty}$, that is invariant under every transformation $g_{S}$. That is, there are some appearancedifferences that remain invariant under every context change under consideration. This seems somewhat surprising, and possibly casts doubt on the plausibility of the assumptions, at least for Case 1.

Case 2. Dimension of $\mathscr{N}_{\infty}=1$.

Proceed as in Case 1, except that $f_{1}, f_{2}, f_{3}$ must be chosen so as to have only multiples of a single vector $\bar{B}_{1}$ in the intersection of their null-components. Obviously, $f_{i}\left(\bar{B}_{1}\right)=\theta$ for $i=1,2,3$ [since the $f_{i}$ map $\mathscr{N}_{\infty}$ into itself, $f_{i}\left(B_{1}\right)$ must be a multiple of $\bar{B}_{1}$, hence must be $\theta$ ], so there are only 6 degrees of freedom in the choice of each $f_{i}$, plus 3 for the choice of $\bar{B}_{1}$, instead of the 27 in Case 1 for the choice of the $f_{i}$. In this case, the general form of $g(\bar{S})$ is given by

$$
g(\bar{S})=h_{S}\left(\bar{A}_{\infty}\right)+g_{1}(\bar{S}) \bar{B}_{1}
$$

where $\bar{A}_{\infty}$ is an arbitrary vector in $\overline{\mathscr{C}}$ and $g_{1}$ is a linear function from $\overline{\mathscr{T}}$ to the real numbers. The choice of $\bar{A}_{\infty}$ involves only two new parameters, since any component along $B_{1}$ cannot affect the values of $g(\bar{S})$, and the choice of $g_{1}$ involves only two additional parameters, since a constant multiple can be absorbed into the specification of $\bar{B}_{1}$. Thus, all the $g_{S}$ are determined by the choice of 25 parameters.

Note that in this case, $f_{S}\left(\bar{B}_{1}\right)=\bar{B}_{1}$; but there is no vector invariant under every $g_{\bar{S}}$. In particular,

$$
\begin{aligned}
g_{S}\left(-\bar{A}_{\infty}\right) & =f_{\bar{S}}\left(-\bar{A}_{\infty}\right)+h_{S}\left(\bar{A}_{\infty}\right)+g_{1}(\bar{S}) \bar{B}_{1} \\
& =-\bar{A}_{\infty}+g_{1}(\bar{S}) \bar{B}_{1} .
\end{aligned}
$$

CASE 3. Dimension of $\mathscr{N}_{\infty}=2$.

In this case, rather than start with $f_{1}, f_{2}$, and $f_{3}$, it is simpler to start by specifying the vectors $\bar{B}_{1}$ and $\bar{B}_{2}$ in $\overline{\mathscr{Q}}$ such that for all $\bar{S}$

$$
\begin{aligned}
& h_{S}\left(\bar{B}_{1}\right)=\theta, \\
& h_{S}\left(\bar{B}_{2}\right)=g_{2}(\bar{S}) \bar{B}_{1}
\end{aligned}
$$

where $g_{2}$ is a linear real-valued function. Now not every $\mathscr{N}_{S}$ can have dimension 3 , for if they all did, one would have $\mathscr{N}_{\infty}=\bar{q}$. Thus, for some $\bar{S}_{1}$, one can choose a nonzero vector $\bar{B}_{3}$ such that $\mathscr{R}_{S_{1}}$ consists of exactly the multiples of $\bar{B}_{3}$. Since by commutativity, every $h_{S}$ maps $\mathscr{R}_{S_{1}}$ onto itself, it follows that there is a real-valued function $k$ defined on $\overline{\mathscr{T}}$ such that for all $\bar{S}$,

$$
h_{S}\left(\bar{B}_{3}\right)-k(\bar{S}) \bar{B}_{\mathbf{3}} \text {. }
$$

From Eq. $5^{\prime \prime}$, one sees that

$$
k(\bar{S}+\bar{T})=k(\bar{S}) k(\bar{T})+k(\bar{S})+k(\bar{T}) .
$$


This functional equation has the general solution

$$
k(\bar{S})=e^{\ell(\bar{S})}-1
$$

where $\ell(\bar{S})$ is an arbitrary linear function from $\overline{\mathscr{P}}$ to the rcals.

The general solution for $g(\bar{S})$ is of the form

$$
g(\bar{S})=h_{S}\left(\bar{A}_{\infty}\right)+g_{1}(\bar{S}) \bar{B}_{1}+g_{2}(\bar{S}) \bar{B}_{2} .
$$

Here, the proportionality constant between $h_{21}$ [the coefficient of $\bar{B}_{1}$ in $h_{S}\left(\bar{B}_{2}\right)$ ] and $g_{2}$ (the coefficient of $\bar{B}_{2}$ above) can be set $=1$, by proper choice of $\bar{B}_{1}$ (otherwise, $\bar{B}_{1}$ would be determined only up to a multiple).

The function $g_{1}$ is given by (14), with $q_{1}=1$ for reasons just mentioned:

$$
g_{1}(\bar{S})=(1 / 2)\left[g_{2}(\bar{S})\right]^{2}+\ell_{1}(\bar{S}) .
$$

The vector $\bar{A}_{\infty}$ may as well be taken $=\bar{B}_{3}$, since any component along $\bar{B}_{1}$ does not affect values of $g(\bar{S})$, while any component along $\bar{B}_{2}$ is mapped by $h_{S}$ into a multiple of $g_{2}(\bar{S}) \bar{B}_{1}$, and can thus be amalgamated with $g_{1}(\bar{S}) \bar{B}_{1}$, since the latter involves an arbitrary linear function $\ell_{1}$. Thus, determination of all the functions $g_{S}$ involves 9 parameters for the choice of $\bar{B}_{1}, \bar{B}_{2}$, and $\bar{B}_{3}, 2$ for the choice of $g_{2}$ (since a constant multiple can be amalgamated with the choice of $\bar{B}_{2}$ ), and 3 each for the choice of linear functions $\ell_{1}$ and $\ell$, a total of 17 . The transformations $f_{S}$ and $g_{S}$ are summarized by the following equations:

$$
\begin{aligned}
f_{S}\left(\bar{B}_{1}\right) & =\bar{B}_{1}, \\
f_{S}\left(\bar{B}_{2}\right) & =g_{2}(\bar{S}) \bar{B}_{1}+\bar{B}_{2}, \\
f_{S}\left(\bar{B}_{3}\right) & = \\
g(\bar{S}) & =\left\{(1 / 2)\left[g_{2}(\bar{S})\right]^{2}+\ell_{1}(\bar{S})\right\} \bar{B}_{1}+g_{2}(\bar{S}) \bar{B}_{2}+\left[e^{\ell(S)}-1\right] \bar{B}_{3}, \\
g_{S}(\bar{A}) & =f_{S}(\bar{A})+g(\bar{S}) .
\end{aligned}
$$

The $\bar{B}_{1}, \bar{B}_{2}$, and $\bar{B}_{3}$ form an interesting coordinate basis for the vector space $\bar{O}$. The choice of a coordinate system corresponding to a special set of primaries is the basic step in any color theory. However, the above basis is not uniquely determined, in the sense that the form of the equations remains unchanged if one adds to $\bar{B}_{2}$ an arbitrary component along $\bar{B}_{1}$ (of course, $\ell_{1}$ would change).

CASE 4. Dimension of $\mathscr{N}_{\infty}=3$.

Choose linearly independent vectors $\bar{B}_{1}, \bar{B}_{2}, \bar{B}_{3}$ in $\bar{Q}$ and linear functions from $\overline{\mathscr{P}}$ to the reals, $g_{3}, \ell_{1}$, and $\ell_{2}$. Define

$$
\begin{aligned}
& g_{2}(\bar{S})=(1 / 2)\left[g_{3}(\bar{S})\right]^{2}+\ell_{1}(\bar{S}), \\
& g_{1}(\bar{S})=(1 / 6)\left[g_{3}(\bar{S})\right]^{3}+g_{3}(\bar{S}) \ell_{1}(\bar{S})+\ell_{2}(\bar{S}) .
\end{aligned}
$$


Now define $f_{S}$ and $g(\bar{S})$ by

$$
\begin{aligned}
f_{S}\left(\bar{B}_{1}\right) & =\bar{B}_{1}, \\
f_{S}\left(\bar{B}_{2}\right) & =g_{3}(\bar{S}) \bar{B}_{1}+\bar{B}_{2}, \\
f_{S}\left(\bar{B}_{3}\right) & =\left[g_{3}(\bar{S})+g_{2}(\bar{S})\right] \bar{B}_{1}+g_{3}(\bar{S}) \bar{B}_{2}+\bar{B}_{3}, \\
g(\bar{S}) & =g_{1}(\bar{S}) \bar{B}_{1}+g_{2}(\bar{S}) \bar{B}_{2}+g_{3}(\bar{S}) \bar{B}_{3},
\end{aligned}
$$

and define $g_{S}(\bar{A})=f_{\bar{S}}(\bar{A})+g(\bar{S})$. This clearly gives all possible solutions to Eqs. 4 and 5 with dimension of $\mathscr{N}_{\infty}=3$, since the coefficients $q_{1}, q_{2}, q_{3}$, in Eqs. 14, 16, and 17 can be taken $=1$ by choosing suitable multiples of $\bar{B}_{1}, \bar{B}_{2}$, and $\bar{B}_{3}$. Thus, the solution depends on 18 parameters. Again, the set of $\bar{B}_{i}$ is not completely determined by the form of these equations, so there is some freedom in choosing such a basis to compare to color-theory primaries.

\section{RELATIONS WITH OTHER THEORIES OF COLOR ADAPTATION AND CONTRAST}

Comparison of the present theory with previous treatments of context effects in perception is most straightforward in the area of color vision. Cross-context matching has been employed frequently in evaluation of adaptation and/or contrast effects on perceived brightness and color. Furthermore, it has long been understood that perceived color depends on the response-levels of 3 physiological response systems, and theories of context effects have been formulated in terms of effects on these systems.

The problems of color adaptation and color contrast cannot be isolated from the more general problems of the temporal and spatial dynamics of the visual system. Ultimately, brightness and color contrast must be incorporated into a theory that encompasses all the interrelated problems of nonhomogeneous stimulation: summation and averaging, inhibition and Mach bands or edge effects, spatial and temporal acuity, contour formation, etc. Similarly, brightness and color adaptation do not merely involve the desensitization of photochemical response systems, but rather, changes in the organization and the noise-level of complex neural systems.

Nevertheless, the present analysis is limited to the case where the appearance of a stimulus element is affected uniformly with respect to space and time by preadapting or surrounding stimuli. The usefulness of such an abstraction lies in the possibility of isolating some information about the 3 fundamental response systems responsible for foveal color vision, and about their interaction and desensitization characteristics. The same abstraction has been made by previous color theorists, with much the same end in view.

The two main theories that I shall discuss are those of von Kries (1905) and of Jameson and Hurvich (1964). In a sense, these theories emphasize opposite aspects 
of the problcm: von Kries' theory is best understood as involving de- or resensitization of fundamental response systems, a multiplicative process, while that of Jameson and Hurvich involves opponent-colors induction, an additive process. Furthermore, the two theories differ in their use of linear versus nonlinear physiological responses.

In terms of the vector space $\overline{\bar{l}}$ introduced in Sec. 8, the von Kries "coefficient law" may be formulated as follows: There exist 3 linearly independent vectors $\bar{A}_{1}, \bar{A}_{2}, \bar{A}_{3}$ in $\overrightarrow{\mathscr{C}}$ such that if $S, T$ overlap, then there exist "coefficients"

$$
g_{1}(S, T), \quad g_{2}(S, T), \quad g_{3}(S, T)
$$

such that

$g_{S, T}\left(p_{1} \bar{A}_{1}+p_{2} \bar{A}_{2}+p_{3} \bar{A}_{3}\right)=p_{1} g_{1}(S, T) \bar{A}_{1}+p_{2} g_{2}(S, T) \bar{A}_{2}+p_{3} g_{3}(S, T) \bar{A}_{3}$.

Since every $\bar{A}$ in $\bar{Q}$ can be written in the form $p_{1} \bar{A}_{1}+p_{2} \bar{A}_{2}+p_{3} \bar{A}_{3}$, Eq. 18 specifies the function $g_{S, T}$ completely. Note also that $g_{S, T}(\theta)=\theta$; that is, the von Kries theory is a special case of the present theory, in which the affine transformations $g_{S . T}$ are linear transformations, possessing a special basis $\bar{A}_{1}, \bar{A}_{2}, \bar{A}_{3}$ with respect to which their matrices are simultaneously diagonal.

Even though the von Kries law falls under Sec. 8, in that the natural additiontransformations are assumed context-invariant, the full theory of Sec. 8 may not be applicable, since there is nothing in the theory that guarantees that Assumption 5 is valid. If Assumptions 5 and 6 hold, then the von Kries theory probably falls under Case 1 of Sec. 8, since $\mathscr{N}_{\infty}$ has dimension 0 unless for some $i(i=1,2,3), g_{i}(S, T)=1$ for all $S, T$, which seems unlikely (see interpretation below).

The physiological interpretation of the von Kries theory is that, for any stimulus element $A$ in $C$, the coefficient of $\bar{A}_{i}$ in the representation of $(\overline{A, \theta})$ is the response-level of the $i$ th (lincar) fundamental response system, and that this $i$ th system is de- or resensitized by a factor $g_{i}(S, T)$ in passing from context $S$ to context $T$, changing the response-level from $p_{i}$ to $p_{i} g_{i}(S, T)$.

If response-functions of the 3 fundamental response systems are unknown, one can try to deduce them by assuming the von Kries law and then trying to find a coordinate system in which the matrices of linear transformations $g_{S, T}$ are simultaneously diagonal.

Modern tests of the von Kries coefficient law have failed to establish any conditions under which it is valid (see Sec. 10), in part perhaps because of the importance of contrast effects in some or all of the conditions under which the theory has been tested. In particular, any theory in which $g_{S, T}$ is a linear transformation predicts that if $A^{T} \equiv B^{S}$, then for any nonnegative $p,(p A)^{T} \equiv(p B)^{S}$. This prediction was proved false, under conditions involving contrast, by Hurvich and Jameson (1957). These authors suggested adding a translation (i.e. making $g_{S, T}(\theta) \neq \theta$ ) to account for opponent-induction effects, thus leading to affine transformations. Affine transformations of $\overline{\mathscr{d}}$ are precisely the type that occur in Sec. 8, although the use of them 
there stems from entirely different considerations, namely, the possible contextinvariance of addition-transformations.

The theory of brightness and color contrast proposed by Jameson and Hurvich (1964) considers only additive (induction) effects on response-levels of the physiological systems. In addition, it assumes that these effects are additive on a neural level where the relation of response-level to stimulus energy is better described by a nonlinear function than by a linear function. This nonlinearity implies that, in fact, the semigroup of addition-transformations of spectral energy distribution functions is not context-invariant. Thus, their theory makes predictions different from those in Sec. 8. Nevertheless, the Jameson-Hurvich equations can be derived from the treatment in Secs. 3-5, and even generalized, by considering a different semigroup of transformations to be context-invariant. The transformations that must be considered are essentially vector increases in the response-levels of the three nonlinear response systems. In the following paragraphs, I shall sketch this application of the theory of secs. 3-5, in an informal and nonrigorous manner, deriving the JamesonHurvich equations as an important special case. However, this derivation of equations does not quite imply that their theory is a special case of the present theory. In fact, the Jameson-Hurvich theory is closely related to the physiological findings of Hartlinc and Ratliff (1958) regarding steady-state interactions in the compound eye of Limulus, and the theory has been applied successfully to steady-state brightness contrast experiments, in which the eye is at least partially adapted to the brightness of each new stimulus element. This sort of partial confounding of stimulus-element and context must be treated with great care.

For convenience, the set $\mathscr{C}$ of stimulus elements is taken to consist of metamerclasses of spectral energy distribution functions. Let the response-levels of the 3 physiological systems, for stimulus-element $A$, seen in context $S$, be denoted

$$
r_{1 S}(A), \quad r_{2 S}(A), \quad r_{3 S}(A) .
$$

They have the following properties:

$$
\begin{array}{ccc}
A^{T} \equiv B^{S} \quad \text { if and only if } \quad r_{i T}(A)=r_{i S}(B), & i=1,2,3, \\
A=B & \text { if and only if } \quad r_{i S}(A)=r_{i S}(B), & i=1,2,3 .
\end{array}
$$

Equation 20 follows from (19) and the fact that $A, B$ denote metamer classes.

Choose some fixed standard context $S$. Let $a=\left(a_{1}, a_{2}, a_{3}\right)$ be any vector of 3 real numbers. Define $A^{a}$ to be the stimulus element (if one exists) such that, for $i=1,2,3$,

$$
r_{i S}\left(A^{a}\right)=r_{i S}(A)+a_{i} .
$$

Denote by $\mathscr{G}$ the set of $a$ such that $A^{a}$ is defined for every $A . \mathscr{G}$ is a commutative semigroup of one-to-one transformations of $C$ (it is a semigroup because if $a, b$ are in $\mathscr{G}$, then $\left(A^{a}\right)^{b}$ is defined for every $A$ ). In practice, one can only require that $A^{a}$ be 
defined for elements $A$ within some "usual range" since at very high levels, the physiological responses will saturate, or injury to the eye is risked. (This aspect is ignored here; a rigorous treatment would involve complex topological reformulation of the theory in Secs. 3-5.) Note that $\mathscr{G}$ at least contains the identity transformation $0=(0,0,0)$, but that, even ignoring the saturation problem mentioned above, not every vector $a$ is in $\mathscr{G}$; for example, it may be impossible to increase the response-level in one system by a large factor without at the same time changing the response level in some other system by some minimum amount. In general, the set of vectors $a$ for which $A^{a}$ is defined for every $A$ (in some "usual range") will depend on the context $S$ chosen as standard for defining the transformations $a$. An important, but plausible assumption is that the semigroup $\mathscr{G}$ is complete with respect to $C$.

Suppose now that Assumptions 1-4 of Sec. 3 are satisfied, so that, in particular, $\mathscr{G}$ is context-invariant with respect to the contexts under consideration. The theory in Secs. 4 and 5 is thus applicable, yielding affine transformations $g_{S, T}$ that describe the effects of shifting from the standard context $S$ to any other context $T$. In this case, however, these transformations can be obtained specifically in terms of the functions $\boldsymbol{r}_{i s}$. First, note that the semigroup operation in $\mathscr{G}$ is just vector addition, since

$$
\begin{aligned}
r_{i S}\left(A^{a b}\right) & =r_{i S}\left(A^{a}\right)+b_{i} \\
& =r_{i S}(A)+a_{i}+b_{i} \\
& =r_{i S}\left(A^{a+b}\right) .
\end{aligned}
$$

Thus, the elements of $\overline{\mathscr{G}}$ are also vectors, and the group operation is vector addition. Examination of the definition of the induced group operation on the stimulus elements shows that it corresponds to vector addition of physiological responses: $r_{i s} \overline{(A, B)}=r_{i s}(A)-r_{i S}(B)$ is well defined and

$$
r_{i s}\left(\bar{A}_{1}+\bar{A}_{2}\right)=r_{i s}\left(\bar{A}_{1}\right)+r_{i s}\left(\bar{A}_{2}\right)
$$

where $\bar{A}_{1}, \bar{A}_{2}, \overline{(A, B)}$, are elements of $\overline{\mathscr{C}}$. From the above, and from Eq. 19, it follows (assuming continuity of $g_{S, T}$ in an appropriate topology) that for $i=1,2,3$,

$r_{i S}\left[g_{S, T}(\bar{A})\right]=g_{i 1}(S, T) r_{1 S}(\bar{A})+g_{i 2}(S, T) r_{2 S}(\bar{A})+g_{i 3}(S, T) r_{3 S}(\bar{A})+g_{i 4}(S, T)$

where $g_{i j}(S, T)$ are the elements of a $3 \times 4$ matrix, depending on $S, T$ but independent of $\bar{A}$.

Note that these results are formally identical with those of Sec. 5 and the beginning of Sec. 8 , since $g_{S, T}$ is represented as an affine transformation in a vector space, but the vector addition is different from that in Sec. 8, taking place on the level of physiological responses rather than stimuli. This comes from the fact that the transformations assumed context-invariant were defined as additions of physiological responses (under context $S$ ), rather than as additions of stimuli. 
Further refinement of Eq. 21 is possible if it is assumed that the stimulus elements can be matched across contexts on each of 3 subjective dimensions, each of which corresponds to equivalence with respect to the response-level of a single physiological process, e.g., $A^{T} \equiv_{i} B^{S}$ if and only if $r_{i T}(A)=r_{i S}(B)$. Suppose that the relations $\equiv_{i}$ are equivalence relations, that $\mathscr{G}$ is context-invariant with respect to these crosscontext matching relations, and that $\equiv \equiv$ is just the conjunction of them. Then the same analysis leads for each $i, i=1,2,3$, to the equation

$$
r_{i s}\left[g_{s, T}(\bar{A})\right]=g_{i i}(S, T) r_{i . S}(\bar{A})+g_{i 4}(S, T) .
$$

These equations are similar to the von Kries coefficient law, in that the $i$ th coordinate of the transformed vector depends on a multiple $g_{i i}(S, T)$ of the $i$ th coordinate of the original one (representing de- or resensitization?), but here there is an additive constant (representing induction?). Also, here, the equations cannot be used to deduce the coordinate system representing physiological responses, without specifying some particular relation between the nonlinear $r_{i s}$ and a linear stage of (photochemical) response. If the responses $r_{i s}$ are assumed to be power functions of some linear stage, then again, one may hope to infer them from cross-context matching experiments.

Finally, in the Jameson-Hurvich theory, the coefficients $g_{i i}$ are assumed to be $=1$, and $g_{i 4}(S, T)$ is assumed to be proportional to the differences in the $i$ th response-level for the context-stimuli $S$ and $T$. This last feature, incidentally, implies that part (ii) of Assumption 5 is satisfied, if the same transformations are applied to context-stimuli as to focal stimulus elements.

\section{TESTS OF CONTEXT-INVARIANCE FOR ADDITION-TRANSFORMATIONS IN COLOR SPACE}

Formally, direct tests of context-invariance for any set of transformations of stimulus elements are quite straightforward: a test is obtained by obtaining matches, under context $S$, of the stimulus complexes $A_{1}{ }^{T}, A_{1}^{a T}, A_{2}{ }^{T}, A_{2}^{a T}$, for different $A_{1}, A_{2}$, with the same $a$ and $T$. Indirect tests are also possible, by testing whether the transformations $g_{S, T}$ are in fact affine. There is an extensive literature on brightness and color contrast, which might lead one to suppose that previous data could be used for such tests. Most of the data, however, suffer in one way or another from confounding of stimulus element changes with context changes. This does not necessarily invalidate such data for the types of analysis envisaged by previous authors, but it does make application of the present theory uncertain. For example, the classic study of Hess and Pretori (1894) confounded stimulus elements with contexts in two ways: first, the matching field was presented simultaneously with the test field, in the same eye, thus constituting an additional, variable context element; and second, the design was a constant-brightness design, in which test-field luminance was changed and then 
context-field luminance was varied until the test-ficld matchcd a fixed match-ficld brightness. The first source of confounding is alleviated in the study by Jameson and Hurvich (1961), by use of successive viewing of the two contexts, but they, too, use a constancy design of a sort, in which they change test-field luminance and surround luminance in a constant ratio (demonstrating that test-field brightness is not necessarily thereby held constant). A similar constancy design was used by Fry and Alpern (1953).

A third type of confounding was mentioned in the previous section; if the eye is allowed to reach a steady state for each new stimulus element, then one aspect of context, adaptive state, changes with changing stimulus elements. This may have been true in the Hess and Pretori study, and is certainly true in the work of Diamond (1953) and Heinemann (1955).

One study of brightness contrast that is apparently free of the above types of confounding is that of Alpern (1953). He studied the context effect called metacontrast, reduction in brightness of a flash of light by a subsequent flash in an adjacent region

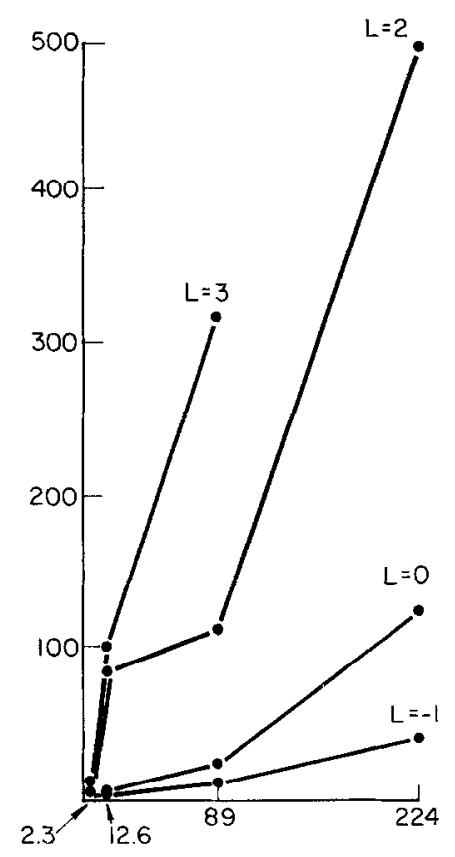

FIG. 2. Functions $g_{S, T}$ for metacontrast, with luminance coordinates. Abscissa is test-field luminance (ft.-lamberts) for test-fields seen in context $S$ (luminance of contrast-field is $1 \mathrm{ft}$.lambert). Ordinate is test-field luminance (ft.-lamberts) for matching test-fields in context $T$ (luminance of contrast-field is $L \mathrm{ft}$.-lamberts). Curves for four different $T$-contexts $(L=1,0,2,3)$ are shown. (Replotted from Alpern, 1953.) 
of the visual field. The matching field, presented in a standard context with no afterflash, was seen by the other eye, in haploscopic viewing, greatly reducing the possibilities of confounding of match-field with context. Direct tests of contextinvariance are not possible, based on his data, but one can attempt to fit an affine equation to his obtained relations between test-field luminances that, in different contexts (different afterflash intensities) match the same match-field. This tests context-invariance of luminance-addition transformations. One can also test contextinvariance of brightness-addition transformations, by taking the cube roots of all luminance values. Affine equations, in one dimension, are simply straight lines. Figures 2 and 3 show some of the data reported by Alpern, replotted in luminance

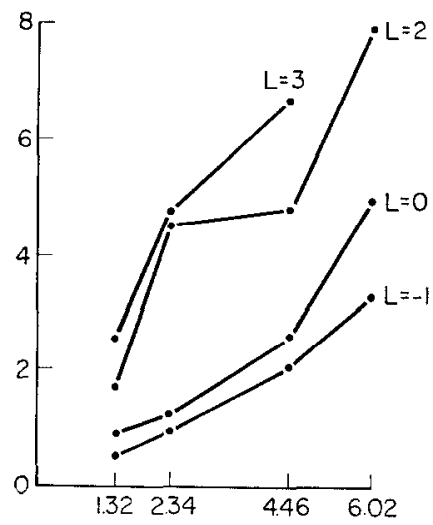

Fig. 3. Functions $g_{S, T}$ for metacontrast, with cube root luminance coordinates. Data are the same as in Fig. 2, but abscissa and ordinate show cube roots of test-field luminances. (Replotted from Alpern, 1953.)

and in cube-root luminance coordinates. The plots are obviously nonlinear but the deviations are not systematic. If one tentatively concludes that neither of the above types of transformation is context-invariant, for metacontrast effects on brightness, this raises the problem of finding what type of transformation, if any, is contextinvariant, for such metacontrast effects.

The most relevant studies of color adaptation and contrast are those of MacAdam (1956) and of Burnham et al. (1957). MacAdam specifically tested the von Kries theory, using haploscopic viewing and a beautifully controlled preadapting field for varying context. He found substantial and systematic deviations in attempting to fit the data by $3 \times 3$ matrices (linear transformations). Perhaps a better fit could be obtained using affine transformations. Burnham $e t$ al. varicd the illumination of a surround-field, obtaining better fits to the color matching data by affine transformations 
than could be obtained with linear ones. These results, together with those of Hurvich and Jameson cited in the previous section, seem to rule out the von Kries theory as having very wide applicability. It may still be appropriate for special types of context effects. The results of Burnham et al. afford positive support for the idea that addition-transformations of energy distributions are context-invariant for certain types of context changes. Their goodness-of-fit is illustrated in Figs. 4 and 5, which show, in the CIE chromaticity diagram, the obtained changes in test-field chromaticity to produce a given match-field color, when the surround is changed from illuminant $C$ to illuminant $A$, and the deviations of obtained from predicted changes, when predictions were made by a least-squares fit of an affine transformation.

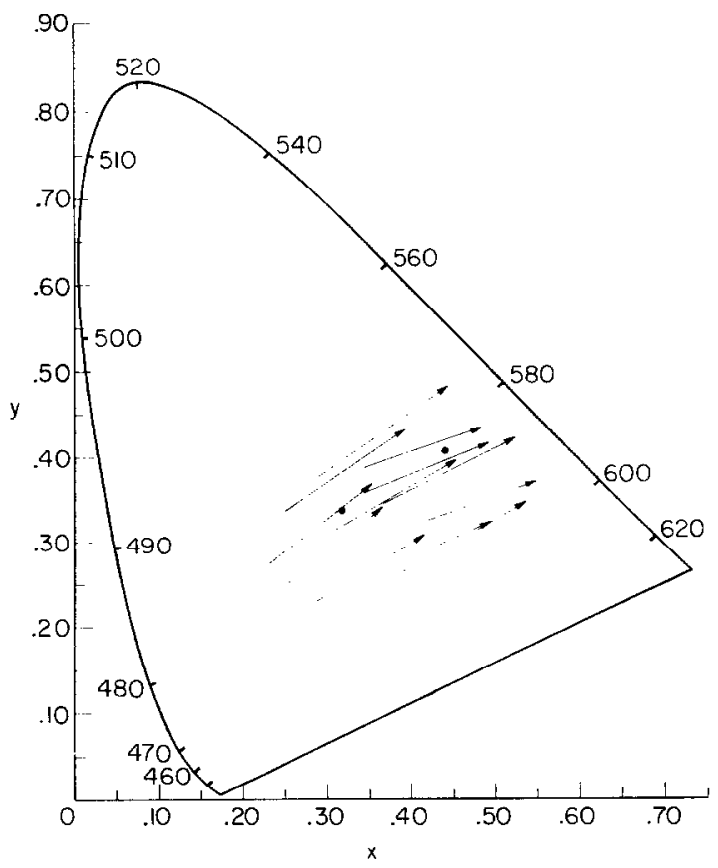

FIG. 4. The CIE chromaticities of the mean matches with illuminant $C$ or $A$ adaptation and surround. Vectors extend from the test-field chromaticity for illuminant $C$ to the test-field chromaticity for illuminant $A$ that produced the same haploscopic match. The axes are CIE chromaticity coordinates $x, y$. 'The chromaticities of the two illuminants are shown as large filled circles. (Adapted from Burnham et al. 1957.) 


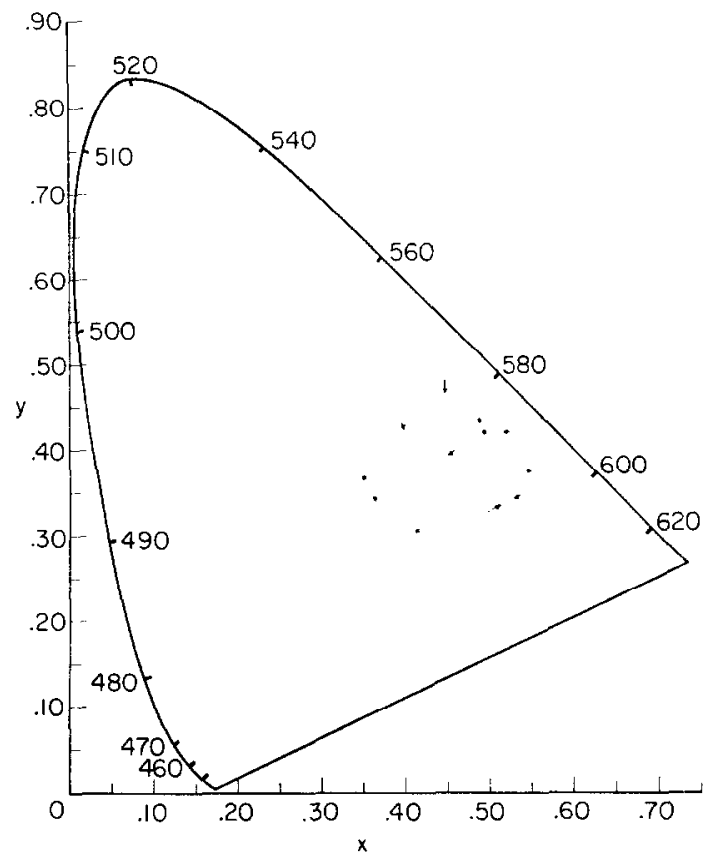

FIG. 5. The CIE chromaticity difference-vectors of obtained versus predicted match-points for illuminant $A$. Obtained match-points are the arrow-points from Fig. 4, while predicted match-points are based on a best-fitting affine transformation (minimized squared error in CIE tristimulus coordinates). Comparisons with color-discrimination data indicate that the deviations are perceptually very small. (Adapted from Burnham et al. 1957.)

\section{REFERENCES}

ALPERn, M. Metacontrast. Journal of the Optical Society of America, 1953, 43, 648-657.

BRINDLey, G. S. Physiology of the retina and the visual pathway. London: Edward Arnold, 1960. Burnham, R. W., Evans, R. M., AND Newhall, S. M. Predictions of color appearance with different adaptation illuminations. Journal of the Optical Society of America, 1957, 47, 35-42.

Diamond, A. L. Foveal simultaneous brightness contrast as a function of inducing- and testfield luminances. Journal of Experimental Psychology, 1953, 45, 304-314.

FRY, G. A., AND ALPERN, M. The effect of a perhipheral glare source upon the apparent brightness of an object. Journal of the Optical Society of America, 1953, 43, 189-195.

HaRTLiNe, H. K., AND RatlifF, F. Spatial summation of inhibitory influences in the eye of Limulus, and the mutual interaction of receptor units. Journal of General Physiology, 1958, 41, 1049-1066.

HeinemanN, E. G. Simultaneous brightness induction as a function of inducing- and test-field luminances. Journal of Experimental Psychology, 1955, 50, 89-96. 
Hess, C., and Pretori, H. Messende Untersuchungen über dic Gesetzmässigkeit des simultanen Helligkeits-Contrastes. Graefes Archiv fuer Ophthalmologie, 1894, 40, 1-24.

Hille, E., and Phillips, R. S. Functional analysis in semigroups. (2nd ed.) American Mathematical Society Colloquium Publication, no. XXXI, 1957.

Hurvich, L. M., AND Jameson, D. Further development of quantified opponent-colour theory. Symposium on visual problems of colour, (National Physical Laboratory, Teddington, England, 1957). Vol. 2, London: H. M. Stationery Office, 1958. Pp. 691-723.

JACOBSON, N. Lectures in abstract algebra. Vol. I. Princeton, New Jersey: Van Nostrand, 1951. JacoBson, N. Lectures in abstract algebra. Vol. II. Princeton, New Jersey: Van Nostrand, 1953. Jameson, D., and Hurvich, L. M. Complexities of perceived brightness. Science, 1961, 133, $174-179$.

Jameson, D., and Hurvich, L. M. Theory of brightness and color contrast in human vision. Vision Research, 1964, 4, 135-154.

IUdD, D. B. Basic correlates of the visual stimulus. In S. S. Stevens (Ed.), Handbook of experimental psychology. New York: Wiley, 1951. Pp. 811-867.

LucE, R. D. Semiorders and a theory of utility discrimination. Econometrica, 1956, 24, 178-191.

MacAdam, D. L. Chromatic adaptation. Journal of the Optical Society of America, 1956, 46, 500-513.

Pontrjagin, L. Topological groups. (Translated from Russian 1st ed.) Princeton, New Jersey: Princeton Univ. Press, 1939.

Stevens, S. S. On the psychophysical law. Psychological Review, 1957, 64, 153-181.

von KrIEs, J. Die Gesichtsempfindungen. In W. Nagel, Handbuch der Physiologie des Menschen. Vol. III. Braunschweig, Germany: Viewig, Frederick und Sohn, 1905. Pp. 205-221.

ZeEMan, E. C. The topology of the brain and visual perception. In M. K. Fort (Ed.), Topology of 3-manifolds. Englewood Cliffs, New Jersey: Prentice-Hall, 1962. Pp. 240-256.

ReCEIVED: June 20, 1966 